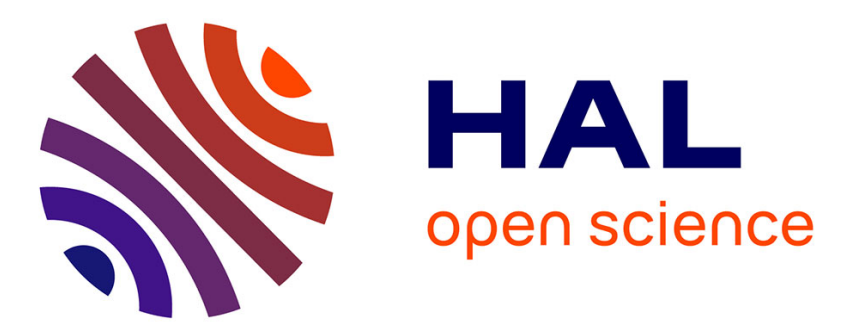

\title{
Compaction Banding in High-Porosity Carbonate Rocks: 2. A Gradient-Dependent Plasticity Model
}

\author{
Youssouf Abdallah, Jean Sulem, Ioannis Stefanou
}

\section{To cite this version:}

Youssouf Abdallah, Jean Sulem, Ioannis Stefanou. Compaction Banding in High-Porosity Carbonate Rocks: 2. A Gradient-Dependent Plasticity Model. Journal of Geophysical Research: Solid Earth, 2020, 125 (12), pp.e2020JB020610. 10.1029/2020JB020610 . hal-03127841

\section{HAL Id: hal-03127841 \\ https://hal.science/hal-03127841}

Submitted on 1 Feb 2021

HAL is a multi-disciplinary open access archive for the deposit and dissemination of scientific research documents, whether they are published or not. The documents may come from teaching and research institutions in France or abroad, or from public or private research centers.
L'archive ouverte pluridisciplinaire HAL, est destinée au dépôt et à la diffusion de documents scientifiques de niveau recherche, publiés ou non, émanant des établissements d'enseignement et de recherche français ou étrangers, des laboratoires publics ou privés. 


\title{
Compaction Banding in High Porosity Carbonate Rocks: 2. A Gradient Dependent Plasticity Model
}

\author{
Youssouf Abdallah $^{1}$, Jean Sulem ${ }^{1}$, and Ioannis Stefanou ${ }^{2}$ \\ ${ }^{1}$ Laboratoire Navier, École des Ponts ParisTech, Univ. Gustave Eiffel, CNRS, \\ Marne-la-Vallée, France \\ ${ }^{2}$ École Centrale de Nantes, Université de Nantes, CNRS, GeM (Institut de Recherche en \\ Génie Civil et Mécanique), Nantes, France
}

Published online on December 2020

\begin{abstract}
To cite:
Abdallah, Y., Sulem, J., \& Stefanou, I. (2020). Compaction banding in high porosity carbonate rocks: 2 . A gradient dependent plasticity model. Journal of Geophysical Research:

Solid Earth, 125, e2020JB020610.

https://doi.org/10.1029/2020JB020610
\end{abstract}




\section{Keypoints}

- The key role of porosity heterogeneity in compaction banding of a weak limestone is modelled using gradient-dependent plasticity.

- The constitutive model is calibrated from triaxial experiments data combined with imaging techniques to compute porosity and strain maps.

- Finite Element simulations can correctly reproduce the dominant features of the compaction bands as observed in the experiments.

\section{Abstract}

Based on the experimental study on Saint-Maximin limestone presented in a companion paper (Abdallah et al., 2020), a gradient-dependent plasticity model is developed to account for the key role of porosity heterogeneity in the formation and propagation of compaction bands. The constitutive law, developed in the frame of a micromorphic continuum theory, contains two hardening variables: the porosity and its second gradient. A calibration method for the additional micromechanical parameters is proposed. Data from the companion paper are used in a four-step calibration procedure. First, the standard Cauchy component is calibrated by means of the macroscopic stressstrain curves. An Asymmetric Cam-Clay (ACC) model is adopted for the yield surface. The dominant wavelength of the porosity heterogeneity of the material is evaluated by applying the Fast Fourier Transformation (FFT) on several porosity profiles obtained on samples before loading. This wavelength is interpreted as the material length that appears in the model. In addition to the deformation maps computed from Digital Volume Correlation (DVC), a method that evaluates the second gradient of porosity is developed to calibrate the hardening laws. A Linear Stability Analysis (LSA) is performed to calibrate the higher order elastic modulus. The constitutive model is implemented in a finite element code and triaxial loading experiments are simulated. The numerical results are consistent with the experimental data in terms of the onset of compaction bands, their thickness and plastic strain. The size effect of the sample is explored and a regular band spacing, comparable to the band thickness, is obtained. 


\section{Introduction}

Studying the formation of compaction bands in sedimentary rocks is of strong interest in rock engineering (Sternlof et al., 2006). These deformation bands may occur due to inelastic compaction induced by several geosystem operations (e.g. geothermal energy (Barbier, 2002); $\mathrm{CO}_{2}$ storage (Rutqvist, 2012); oil and gas production (Olsson et al., 2002); see also Holcomb et al. (2007)). In addition, these bands can be interpreted as indicators of the stress state history in a geological formation. The observations performed on natural fields and on laboratory tested samples show that these bands are likely to form in high-porosity rocks (Rath et al., 2011; Baud et al., 2017). Furthermore, although these bands are observed to form in sandstones as well as in carbonate rocks, it appears that the micro-mechanisms involved in strain localization are not similar for the two rock types (Zhu et al., 2010; Cilona et al., 2012).

Modelling compaction bands has been addressed in a number of papers over the last decades (e.g. Rudnicki and Rice (1975); Issen and Rudnicki (2000); Rudnicki (2007); Das et al. (2011); Stefanou and Sulem (2014)). It is well known that standard constitutive models developed in the frame of Classical Cauchy continuum theory are unable to assess the thickness of the deformation bands. This can be tracked to the absence of an internal length linked to the microstructure of the material in the formulation of the constitutive relationships. The experimental observations performed in-situ and in laboratory show that the deformation band thickness is scaled by the grain size (Du Bernard et al., 2002; Sulem and Ouffroukh, 2006; Cilona et al., 2014). To overcome this limitation, macroscopic constitutive laws can be enriched by introducing material lengths that account for the microstructure (Vardoulakis and Sulem, 1995; Bésuelle and Rudnicki, 2004). However, the difficulty lies in the calibration of the additional parameters introduced in such formulations.

In classical constitutive models, the material parameters are calibrated using experimental laboratory tests such as triaxial, oedometric, plane strain loading experiments, etc., assuming that the samples are loaded homogeneously during such experiments. However, the additional parameters introduced in micromorphic media (i.e. continua with microstructure) require the development of new calibration procedures, which are able to capture the transition from homogeneous to inhomegeous deformation and to assess

the role of microstructure heterogeneities. For this purpose, global measure- 
ments of stresses, strains and other state variables are not sufficient, and local measurements at the scale of the microstructure are required. In the last two decades, several imaging and post-processing techniques, which enable data collection at the micro-scale level, have been developed (Hall et al., 2012). Nonetheless, few works have been dedicated to the calibration of higher order continua based on local measurements (e.g. for Cosserat continuum: Wang et al. (2016); Esin et al. (2017)). A back analysis of the thickness of deformation bands as observed in element tests can give access to the material length of the underlying constitutive model (e.g. Raude et al. (2015)). However, this procedure poorly constrains the calibrated higher order parameters since other model parameters influence the deformation band thickness and the slope of the softening branch of the stress-strain curve. Therefore, full field measurements based on advanced imaging techniques and digital volume correlation appear to be appropriate for robust calibration of higher order continuum models.

The objective of this study is to propose a constitutive model that can describe the formation of compaction bands in high-porosity carbonate rocks and a reliable method that permits to calibrate the additional parameters. The experimental data presented in the companion paper (Abdallah et al., 2020) are used to calibrate the proposed model. In Section 2, a gradientdependent plasticity model is developed based on the non-local aspect of the deformation mode observed in the experiments. Then, the model is interpreted in the frame of the micromorphic continuum theory. A full calibration procedure based on the experimental results (macroscopic mechanical data, porosity and deformation maps) is presented thereafter in Section 3. Finally, Section 4 is devoted to the implementation of the proposed constitutive law in the Finite Element code Numerical geolab (Stefanou, 2018) and to the numerical simulation of compaction banding. The formation and evolution of compaction bands are simulated in due course of loading. The size effect is also studied by considering samples with different sizes. A comparison between the numerical results and the experimental data is done in order to test the proposed model. 


\section{Gradient-dependent Plasticity}

\section{$2.1 \quad$ Experimental Observations}

The effect of the porosity heterogeneity on compaction banding in highporosity limestones has been experimentally explored in Abdallah et al. (2020). It is shown that the Saint-Maximin limestone exhibits a porosity fluctuation from about $30 \%$ to more than $40 \%$ at the scale of one centimeter. This heterogeneity of the porosity is related to a heterogeneity of interparticle cementation. Despite this fluctuation, a good repeatability of the experiments has been obtained allowing to assume that samples of $4 \mathrm{~cm}$ in diameter and $8 \mathrm{~cm}$ in height can be considered as representative elementary volumes. The experimental observations have shown that the porosity heterogeneity is responsible for the compaction bands nucleation and propagation, since these bands are identified in high-porosity zones, whereas denser zones remain undeformed. In addition, several large pores (i.e. large in comparison to the pore and grain sizes) do not collapse even under relatively high stresses (Figure 1), as opposed to other studies showing that larger pores collapse first (e.g. Zhu et al. (2010)). These pores lie in low-porosity zones and are protected by a surrounding stiffer matrix of well-cemented and large grains. It is worth to mention that no compaction fronts propagating from the boundaries toward the center of the sample have been observed in the experiments, since special attention was devoted to minimize the friction at the interface between the sample and the loading platens (details can be found in the companion paper (Abdallah et al., 2020)). Thus, the modelling approach in this study will focus on the effect of the local porosity heterogeneity on compaction banding (unlike the modelling approach proposed by Shahin and Buscarnera (2020), who studied the effect of frictional boundaries). An "equivalent homogeneous" continuum containing a material length that accounts for the length scale of the porosity heterogeneity is thus introduced.

\subsection{Non-local Approach}

The experimental observations made in the companion paper (Abdallah et al., 2020), suggest that the deformation state at a point does not only depend on the local properties, but also on the properties of the material at the vicinity. This "non-local" character of the response can be described by 


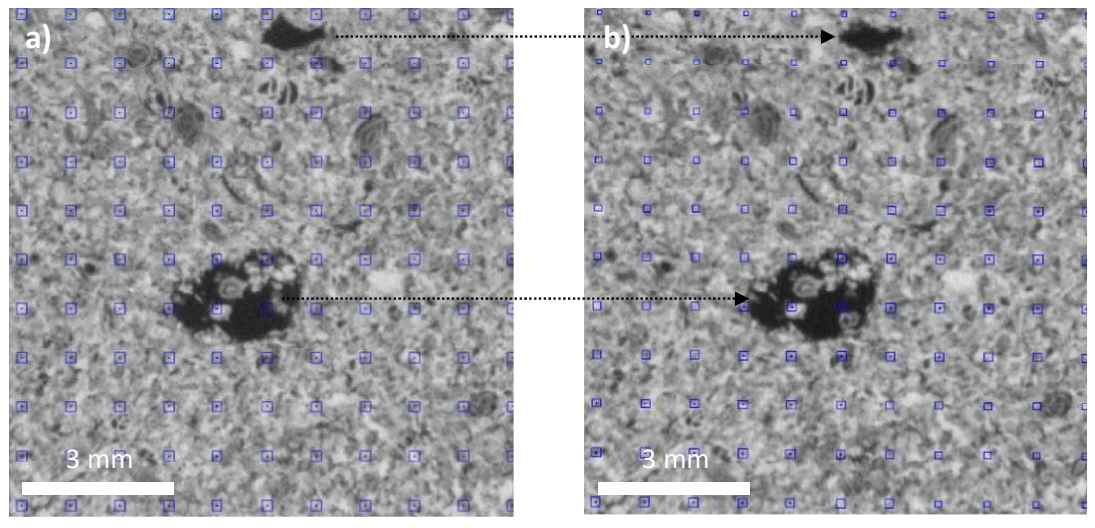

Figure 1: Undeformed large pores in a low-porosity zone: (a) at the initial state; (b) after loading (Abdallah et al., 2020).

resorting to non-local plasticity models, in which the plastic hardening variables are averaged over a subvolume centered at the material point (Bésuelle and Rudnicki, 2004). A simple way to represent the averaged quantities is obtained by developing the Taylor expansion of the plastic variable up to the second order and to consider a subvolume of cubic shape. In this case, the plastic hardening parameter $\kappa$ and its second gradient $\nabla^{2} \kappa$ are considered as two independent variables and a gradient-dependent plasticity model is therefore constructed (De Borst and Mühlhaus, 1992; Pamin, 1996). The yield surface $F$ is expressed in terms of $F\left(\sigma_{i j}, \kappa, \nabla^{2} \kappa\right)$, where $\sigma_{i j}$ is the stress tensor. The side length of the subvolume - referred henceforth by $L$ represents a material length in the constitutive law, which permits to obtain deformation bands of finite thickness as opposed to classical plasticity models for which strain localizes on a mathematical plane (Vardoulakis and Sulem, 1995).

Compaction bands are associated with porosity reduction (Rath et al., 2011) induced either by grain crushing as in the case of the Saint-Maximin limestone (Abdallah et al., 2020) or by other micro-mechanisms such as grain re-arrangement (Cashman and Cashman, 2000), calcite grain twinning (Cilona et al., 2014), pore collapse (Zhu et al., 2010) and pressure solution (Rustichelli et al., 2012). Hence, the plastic porosity change $\phi^{p l}$ is taken here as the plastic hardening parameter $\kappa$. In addition, assuming that the deformation of the solid matrix is negligible, the plastic porosity change can be approximated by the plastic volumetric strain $\epsilon_{v}^{p l}$ (Coussy, 2011). The 
validity of this assumption in view of the experimental data will be discussed later in Section 3.3.2. Consequently, $\kappa \equiv \epsilon_{v}^{p l}$ and $F=F\left(\sigma_{i j}, \epsilon_{v}^{p l}, \nabla^{2} \epsilon_{v}^{p l}\right)$. The incremental constitutive equation is:

$$
\dot{\sigma}_{i j}=\left(C_{i j k l}^{e}-\langle 1\rangle \frac{b_{i j}^{g} b_{k l}^{f}}{H_{1}}\right) \dot{\epsilon}_{k l}-\langle 1\rangle \frac{b_{i j}^{g} \frac{\partial F}{\partial \nabla^{2} \epsilon_{v}}}{H_{1}} \nabla^{2} \dot{\epsilon}_{v}
$$

where $\dot{\sigma}_{i j}$ is the rate of the stress tensor, $\dot{\epsilon}_{k l}$ is the rate of the strain tensor, $\nabla^{2} \dot{\epsilon}_{v}$ is the rate of the second gradient of the volumetric strain, $C_{i j k l}^{e}$ is the elasticity tensor, $H_{1}=b_{m n}^{f} \frac{\partial g}{\partial \sigma_{m n}}-\frac{\partial F}{\partial \epsilon_{v}^{p l}} \frac{\partial g}{\partial p}, p$ is the mean stress, $b_{k l}^{f}=\frac{\partial F}{\partial \sigma_{m n}} C_{m n k l}^{e}$, $b_{i j}^{g}=C_{i j m n}^{e} \frac{\partial g}{\partial \sigma_{m n}}, g$ is the plastic potential, $(i, j, k, l, m, n)$ are tensor indices which can take integer values from 1 to 3 following the Einstein notation and $\langle 1\rangle$ is the Macaulay brackets equal to 1 in the plastic regime (if $F=0$ and the plastic multiplier $\dot{\lambda}>0$ ) and equal to 0 in the elastic domain (if $F<0$ or if $F=0$ and $\dot{\lambda} \leq 0$ ). The mathematical development to obtain Equation 1 is presented in A.

\subsection{Gradient-dependent Plasticity Interpreted as a Mi- cromorphic Continuum}

The gradient-dependent plasticity model can be interpreted as a micromorphic continuum. In such continua, a subvolume is attached to each material point leading to introduce additional degrees of freedom (Mindlin, 1964). A rigorous mathematical framework is developed in this section for a general micromorphic continuum theory following the work of Germain (1973) which permits to define the equilibrium equations, boundary conditions and material parameters. In addition, the required restrictions to reach the gradientdependent plasticity model developed in Section 2.2 are introduced.

\subsubsection{Equilibrium Equation}

In a micromorphic continuum, the kinematics of deformation of the subvolume attached to each material point is described from the displacement vector $u_{i}$ and its first and higher-order gradients $\left(\chi_{i j}, \chi_{i j k}, \chi_{i j k l}, \ldots\right)$, where all these quantities are considered as independent fields. Since the second gradient of strain appears in the constitutive law (Equation 1), the required fields are $u_{i}, \chi_{i j}$ and $\chi_{i j k}$. To match with the second gradient theory, the microdeformation and its gradient are identified with the deformation of the 
(macro-) continuum and its gradient: $\chi_{i j} \equiv u_{i, j}$ and $\chi_{i j k} \equiv u_{i, j k}$. The internal power density $\delta w$ is finally written in the following form, where the notation ${ }_{, j}$ represents the gradient operator (details of the mathematical developments can be found in B):

$$
\delta w=\sigma_{i j} u_{i, j}+\nu_{i j k} u_{i, j k}+\zeta_{i j k l} u_{i, j k l}
$$

The introduced dual quantities are: $\sigma_{i j}$ which is the standard Cauchy stress, $\nu_{i j k}$ and $\zeta_{i j k l}$ which are higher order stresses. For simplicity, the body forces are neglected. Hence, the principle of virtual power gives:

$$
\int_{\mathscr{D}}\left[\sigma_{i j} u_{i, j}+\nu_{i j k} u_{i, j k}+\zeta_{i j k l} u_{i, j k l}\right] d v=\int_{\partial \mathscr{D}}\left[T_{i} u_{i}+N_{i} D u_{i}+P_{i} D^{2} u_{i}\right] d s
$$

where $T_{i}, N_{i}$ and $P_{i}$ are the contact forces dual to $u_{i}$, its first and second normal derivatives $D u_{i}$ and $D^{2} u_{i}$ with respect to the boundary $\partial \mathscr{D}$ of the domain $\mathscr{D}$ (see Germain (1973)). From the volume integral in Equation 3, one derives the following equilibrium equation for the Cauchy stress and the higher order stresses:

$$
\left(\sigma_{i j}-\nu_{i j k, k}+\zeta_{i j k l, l k}\right)_{, j}=0
$$

Note that, by omitting the highest order term $\zeta_{i j k l}$, the equilibrium equation degenerates to the one of the second gradient theory as defined by Germain (1973).

The enhanced kinematics considered in the first grade micromorphic continuum of degree two introduces 36 additional degrees of freedom: 9 components for $\chi_{i j}$ and 27 for $\chi_{i j k}$. However, the gradient-dependent constitutive law formulated in Section 2.2 introduces only one additional degree of freedom, which is the second gradient of the volumetric strain $\nabla^{2} \epsilon_{v}$. Thus, some assumptions must be set in order to restrict the general micromorphic model to the considered gradient model. Raude et al. (2015) have presented the formulation of a "second gradient dilation model", where the only additional degree of freedom is the first gradient of the volumetric strain $\nabla \epsilon_{v}$ (see also Forest and Sievert (2006)). Here, a similar approach is presented, but for a "third gradient dilation model". To do so, only the volumetric quantities of the higher-order stresses and of the kinematic variables are considered:

1. $\nu_{i j k}=\delta_{i j} \nu_{k}$ and $\chi_{i j}=\frac{1}{3} \delta_{i j} \chi$ 


$$
\text { 2. } \zeta_{i j k l}=\delta_{i j} \delta_{k l} \zeta \text { and } \chi_{i j k}=\frac{1}{3} \delta_{i j} \chi_{k}
$$

where $\delta_{i j}$ is the Kronecker symbol. The internal work density expression is simplified to:

$$
\delta w=\sigma_{i j} u_{i, j}+\nu_{k} \chi_{, k}+\zeta \chi_{k, k}
$$

where $\chi_{k, k}$ is the second gradient of the volumetric strain: $\chi_{k, k}=u_{i, i k k}=$ $\nabla^{2} \epsilon_{v}$. Moreover, the equilibrium equation becomes:

$$
\tilde{\tau}_{i j, j}=0
$$

where $\tilde{\tau}_{i j}=\sigma_{i j}-\delta_{i j} \nu_{k, k}+\delta_{i j} \delta_{k l} \zeta_{, l k}$ is the so-called "true" stress tensor (Vardoulakis and Sulem, 1995).

\subsubsection{Boundary Conditions}

The surface integral in Equation 3 permits to write the boundary conditions:

$$
\begin{gathered}
T_{i}=\tilde{\tau}_{i j} n_{j}+D_{p} n_{p}\left(\nu_{k}-\zeta_{, k}\right) n_{k} n_{i}-D_{i}\left(\left(\nu_{k}-\zeta_{, k}\right) n_{k}\right)+D_{p} n_{p} D_{s} n_{s} \zeta n_{i} \\
-D_{p} n_{p} D_{k}\left(\zeta n_{k}\right) n_{i}-D_{i}\left(D_{p} n_{p} \zeta-D_{k}\left(\zeta n_{k}\right)\right)+D_{p}\left(\zeta D_{i}\left(n_{p}\right)\right) \\
N_{i}=\left(\nu_{k}-\zeta_{, k}\right) n_{k} n_{i}+2 D_{p} n_{p} \zeta n_{i}-D_{k}\left(\zeta n_{k}\right) n_{i}-D_{i} \zeta \\
P_{i}=\zeta n_{i}
\end{gathered}
$$

where $n_{i}$ is the unit outward normal vector to the boundary $\partial \mathscr{D}$ and the tangential derivation $D_{j}$ is introduced following the definition of Mindlin (1964) (see C). Considering that we are interested in modelling strain-localization inside the body and not boundary-layer effects at an interface boundary, it is assumed that the higher order contact forces $N_{i}$ and $P_{i}$ vanish. The aforementioned assumptions result in $\zeta=0$ and $\nu_{k}=\zeta_{, k}$ on the boundary. In this case, the surface traction is given by the standard Cauchy condition $T_{i}=\tilde{\tau}_{i j} n_{j}=\sigma_{i j} n_{j}$.

\subsubsection{Constitutive Relationships}

To rewrite the constitutive law given in Equation 1, the yield surface is assumed to depend on the Cauchy stress, the scalar stress $\zeta$ and the hardening parameter $\epsilon_{v}^{p l}$ :

$$
F\left(\sigma_{i j}, \nu_{k}, \zeta, \epsilon_{v}^{p l}\right)=0
$$




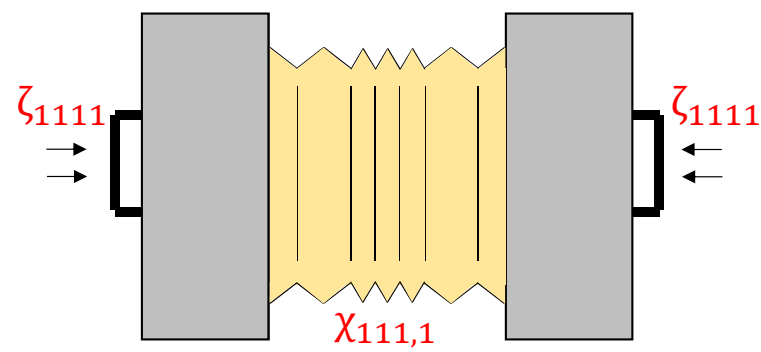

Figure 2: Higher order stress $\zeta_{1111}$ and second gradient of strain $\chi_{111,1}$ : the "accordion effect".

In addition, isotropic linear elasticity is assumed for the higher-order stresses:

$$
\dot{\nu}_{k}=A L^{2} \dot{\chi}_{, k}
$$

and

$$
\dot{\zeta}=B L^{4} \dot{\chi}_{k, k}
$$

where $A$ and $B$ are additional higher-order elastic moduli and $L$ is a material length. Since the first gradient of the volumetric strain is unnecessary in the developed gradient-dependent plasticity model (Section 2.2), the modulus $A$ is put equal to zero. The consistency equation $\dot{F}=0$ leads to the following constitutive law:

$$
\dot{\sigma}_{i j}=\left(C_{i j k l}^{e}-\langle 1\rangle \frac{b_{i j}^{g} b_{k l}^{f}}{H_{1}}\right) \dot{\epsilon}_{k l}-\langle 1\rangle \frac{b_{i j}^{g} \frac{\partial F}{\partial \zeta} B L^{4}}{H_{1}} \dot{\chi}_{k, k}
$$

which is equivalent to the gradient-dependent plasticity constitutive law given in Equation 1, since $\frac{\partial F}{\partial \zeta} B L^{4}=\frac{\partial F}{\partial \nabla^{2} \epsilon_{v}}$ and $\chi_{k, k}=u_{i, i k k}=\nabla^{2} \epsilon_{v}$. The dependence of the yield surface on the higher-order stress state $\zeta$ is defined once its evolution with the second gradient of the volumetric strain is characterized. Following Mindlin (1964), one can illustrate the higher order stress $\zeta_{1111}$ and the second gradient of strain $\chi_{111,1}$ as shown in Figure 2 with the "accordion" effect. The deformation of the subvolume is concentrated at the center.

\section{Calibration Procedure}

The constitutive law of the gradient-dependent plasticity model given in Equations 12 and 13 can be seen as an extension of a constitutive law in 
the standard Cauchy formulation. The calibration of this enhanced model is done in several steps illustrated in Figure 3, where the data of the samples tested under different confining pressures from Abdallah et al. (2020) are used. A full characterization of this model requires a calibration of:

1. The Cauchy continuum component, where the standard parameters are calibrated using the macroscopic mechanical data averaged over the rock sample.

2. The material length $L$, which is evaluated by means of the fast Fourier transformation, applied on the porosity map computed in the initial state.

3. The dependence of the yield surface on the second gradient of porosity, which is considered as an internal variable, or equivalently on the higher order stress: $\frac{\partial F}{\partial \zeta}$. In this step, $\nabla^{2} \phi$ is evaluated from the porosity maps computed at several states of deformation. Furthermore, the mean plastic volumetric strain is calculated from the Digital Volume Correlation (DVC) maps.

4. The higher order elastic modulus $B$, which is assessed by means of a linear stability analysis performed on stress states on the initial yield surface. The range of confining pressures, for which compaction bands occur at the plasticity onset in axisymmetric triaxial loading, permits to evaluate the modulus $B$.

Each of the following subsections is devoted to one of the steps stated above.

\subsection{Calibration of the Cauchy Component}

Based on the stress and strain measurements at the scale of the samples, a standard calibration of the Cauchy parameters is performed:

\subsubsection{Elastic Parameters}

An isotropic behavior is assumed for the elastic regime, which can be characterized by two parameters: the Young modulus $E$ and the bulk modulus $K$. In absence of data on unloading/reloading cycles, the elastic moduli are simply evaluated on the initial linear part of the stress strain curves, as the 


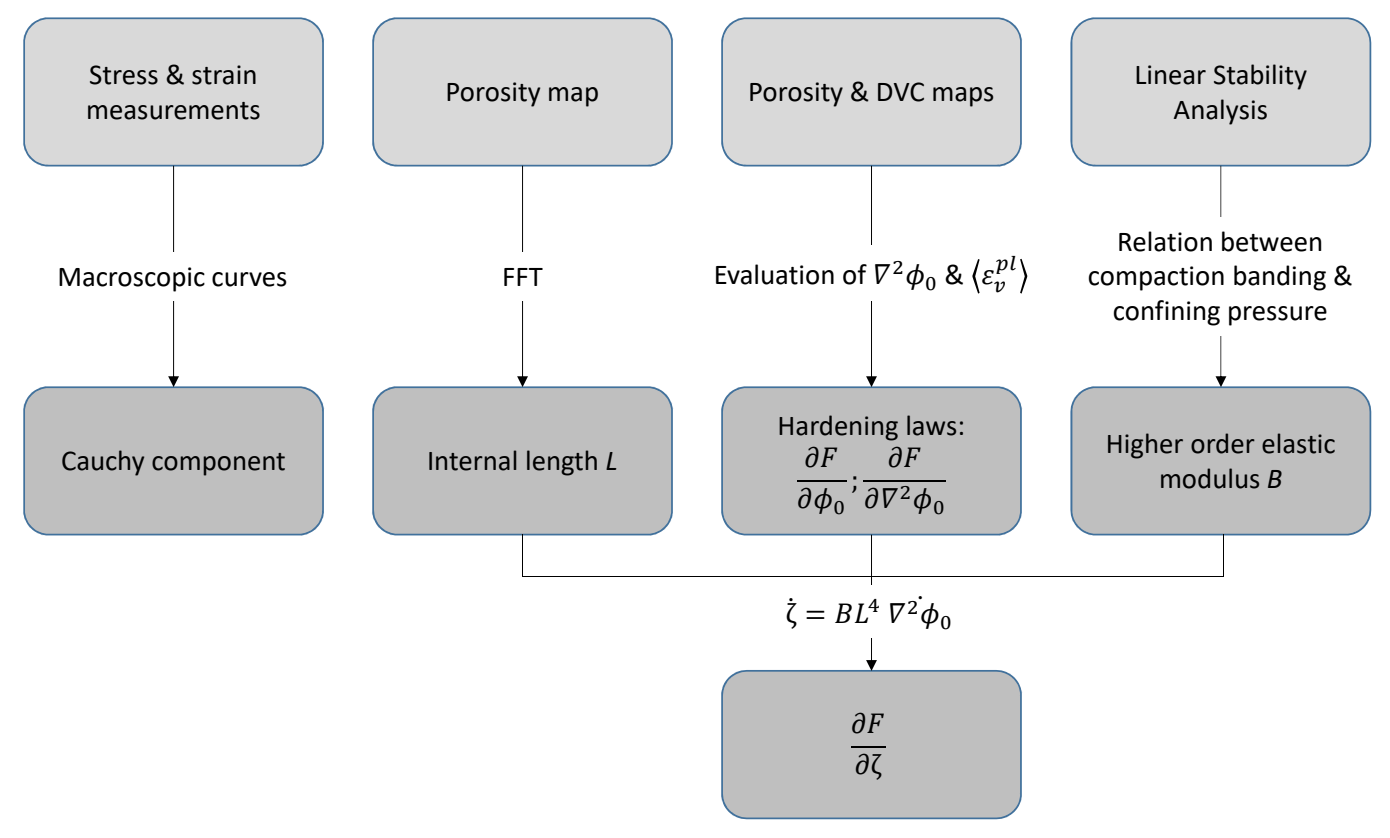

Figure 3: Calibration procedure of the gradient-dependent plasticity model.

mean stress is expressed in terms of the elastic volumetric strain as $\dot{p}=K \dot{\epsilon}_{v}^{e l}$ and the deviatoric stress in axisymmetric triaxial compression is expressed in terms of the elastic axial strain as $\dot{q}=-\frac{E}{3} \dot{\epsilon}_{a}^{e l}$. The calibrated values are: $E=6230 \mathrm{MPa}$ and $K=2810 \mathrm{MPa}$ (Figure 4).

\subsubsection{Initial Yield Surface}

The Asymmetric CamClay (ACC) model (Samudio, 2017) is considered for the yield surface shape. A presentation of this model is given in the D. The yield surface is expressed as:

$$
F=q^{2} e^{k x}+M^{2}\left(p-p_{c}\right)\left(p+p_{t}\right)
$$

where $k$ is the hardening-softening threshold, $p_{c}$ and $p_{t}$ are the compression and traction isotropic yield stresses and $x=\frac{2 p+p_{t}-p_{c}}{p_{t}+p_{c}}$ is the normalized mean stress with respect to the isotropic axis domain size. In order to characterize the yield surface, four parameters must be calibrated: $k, M, p_{t}$ and $p_{c}$. Results are given in Table 1 and the corresponding yield surface is plotted together with the yield stresses in Figure 5a. 
a)

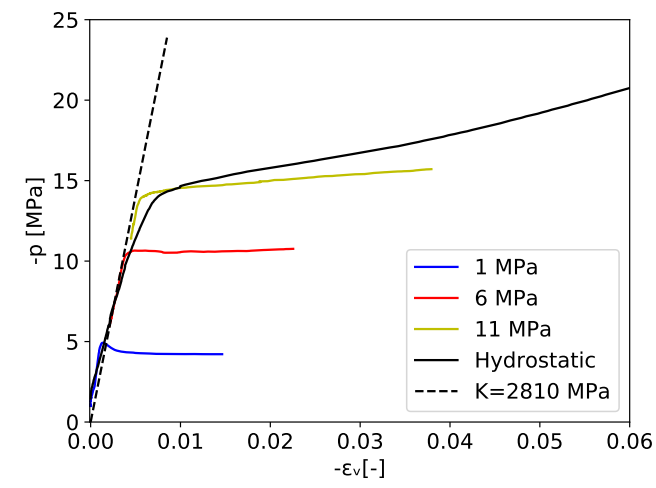

b)

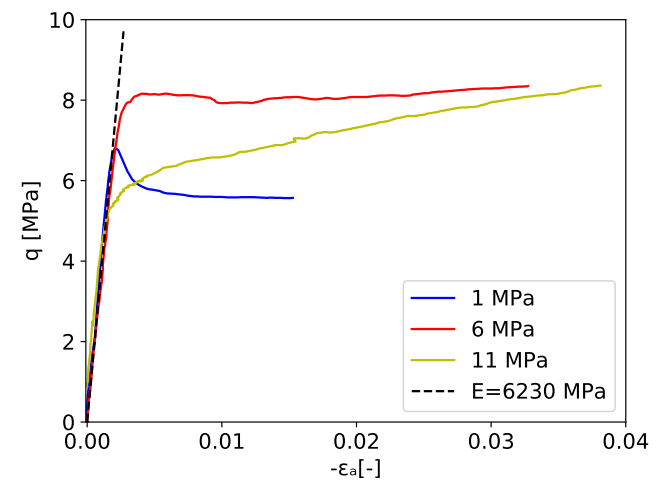

Figure 4: Calibration of the elastic parameters. Curves are collected from the companion paper (Abdallah et al., 2020).

Table 1: Calibrated parameters of the initial yield surface.

\begin{tabular}{cccc}
\hline$k[-]$ & $M[-]$ & $p_{c}[\mathrm{MPa}]$ & $p_{t}[\mathrm{MPa}]$ \\
\hline-0.7 & 0.92 & -15.4 & -0.7 \\
\hline
\end{tabular}

a)

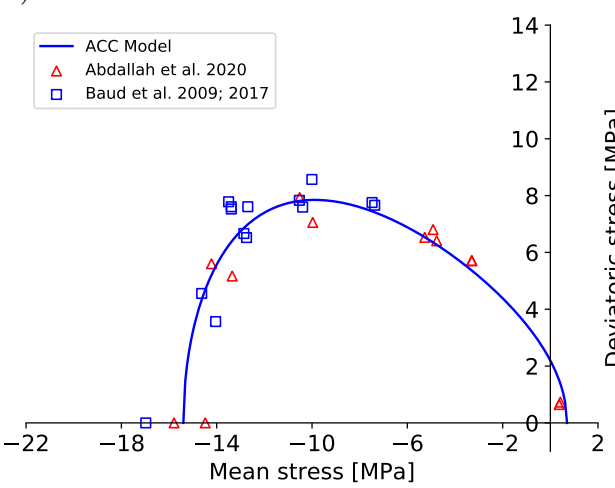

b)

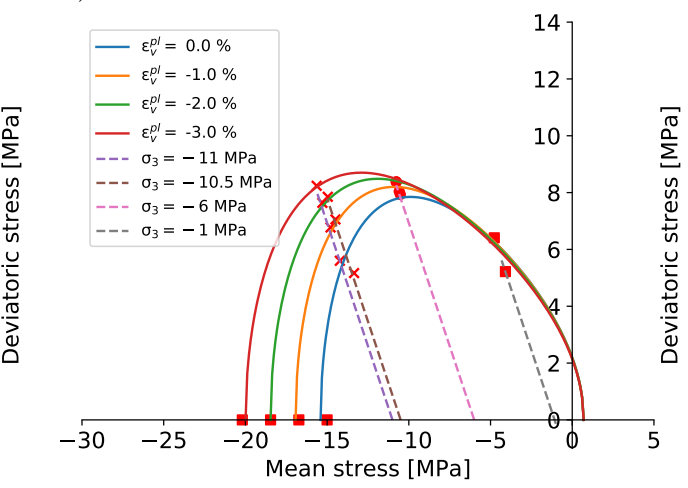

Figure 5: (a) Initial ACC yield surface fitted to the yield stress data: red triangles represent data from Abdallah et al. (2020) \& blue squares for Baud et al. (2009); Baud et al. (2017) data; (b) Yield surface evolution calibrated on the stress states for several confining pressures and at different levels of hardening. 


\subsubsection{Yield Surface Evolution}

Hardening is observed in hydrostatic loading experiments and in triaxial experiments at relatively high-confining pressures. On the other hand, a slight softening is observed at low confining pressures, whereas a perfectly plastic behavior occurs in the transitional regime (Figure 4). To describe these stress-dependent hardening/softening behaviors and to model the yield surface evolution, the two variables $p_{c}$ and $M$ are simply assumed to linearly evolve with the plastic internal variable $\epsilon_{v}^{p l}$ (Equation 15), whereas the variables $p_{t}$ and $k$ are assumed to remain constant. An increase of the parameter $p_{c}$ (in absolute value) is responsible for a global hardening, whereas a decrease of the parameter $M$ induces a softening.

$$
\begin{aligned}
& \dot{p}_{c}=h_{1} \dot{\epsilon}_{v}^{p l} \\
& \dot{M}=h_{2} \dot{\epsilon}_{v}^{p l}
\end{aligned}
$$

For each experiment, the stress state is evaluated at several levels in the plastic regime. Then, the hardening variables are calibrated as shown in Figure 5b. The obtained values are: $h_{1}=1.53 \times 10^{2} \mathrm{MPa}$ and $h_{2}=4.21$. Although the softening behavior is slightly underestimated by the calibrated model, the hardening laws give a very good representation for hydrostatic loading and for intermediate/high confinement triaxial tests. To improve the calibration, an evolution of one additional variable $\left(p_{t}\right.$ or $\left.k\right)$ can be considered. However, since modelling compaction bands is the objective of this study, the proposed hardening laws are sufficient.

\subsubsection{Plastic Potential}

A simple Drucker-Prager plastic potential is considered:

$$
g=\beta p+q
$$

From the flow rule $\left(\dot{\epsilon}_{v}^{p l}=\dot{\lambda} \frac{\partial g}{\partial p} ; \dot{\gamma}^{p l}=\dot{\lambda} \frac{\partial g}{\partial q}\right)$, the dilatancy parameter $\beta$ links the rate of the plastic volumetric strain to the rate of the plastic shear strain magnitude: $\beta=\frac{\dot{\epsilon}_{v}^{p l}}{\dot{\gamma}^{p l}}$. This parameter is calibrated on the experimental data as shown in Figure 6. A constant dilatancy parameter, independent of the stress state and equal to -0.75 , fits well the data. 


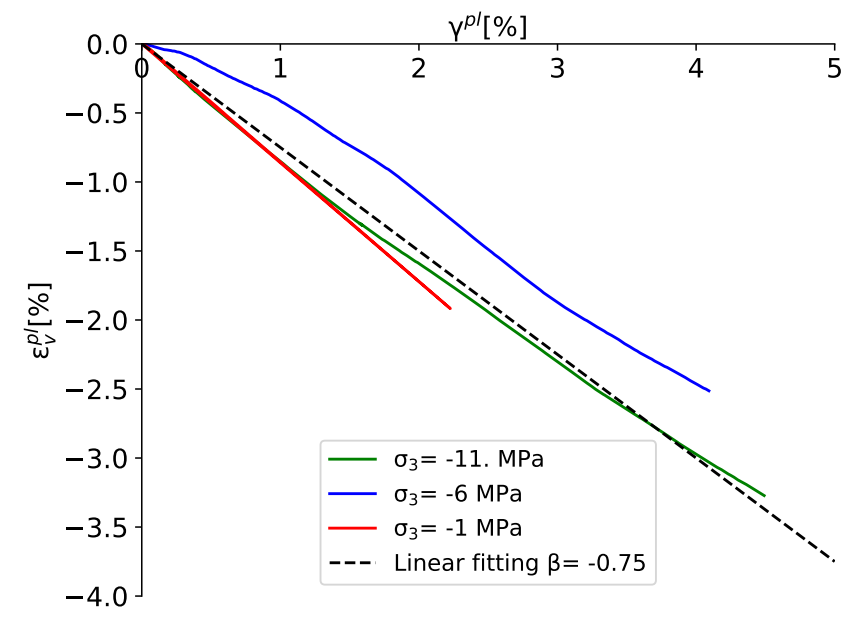

Figure 6: Plastic volumetric strain vs plastic shear strain magnitude measured on 3 samples tested at different confining pressures. The dashed line represents the model prediction for $\beta=-0.75$.

\subsection{Material Length Calibration}

As discussed in Section 2.1, porosity heterogeneity is found to control compaction banding in high-porosity limestones. The computed porosity maps at the initial state of several Saint-Maximin limestone samples exhibit an alternation between low- and high-porosity zones, whose mean values are $35 \%$ and $42 \%$ respectively (Abdallah et al., 2020). Therefore, the porosity heterogeneity is schematically represented as a periodic pattern of low- and high-porosity cubic zones as shown in Figure 7. An elementary cell with side length $L^{\prime}$ centered over a high-porosity zone is defined as the primitive cell of the periodic lattice. In the frame of the gradient-dependent plasticity, this primitive cell is considered as the representative elementary volume over which the porosity and its second gradient are evaluated and over which compaction banding is studied. In other words, the material length $L$ introduced in the constitutive law (see Section 2.2) is identified here with the dominant wavelength of the porosity heterogeneity $L \equiv L^{\prime}$. It is worth to mention that a natural material like Saint-Maximin limestone exhibits porosity heterogeneity at various scales. We are interested here in capturing the most developed compaction bands inside the sample and in seeking the relevant material length that controls the thickness of the observed bands. Therefore, 


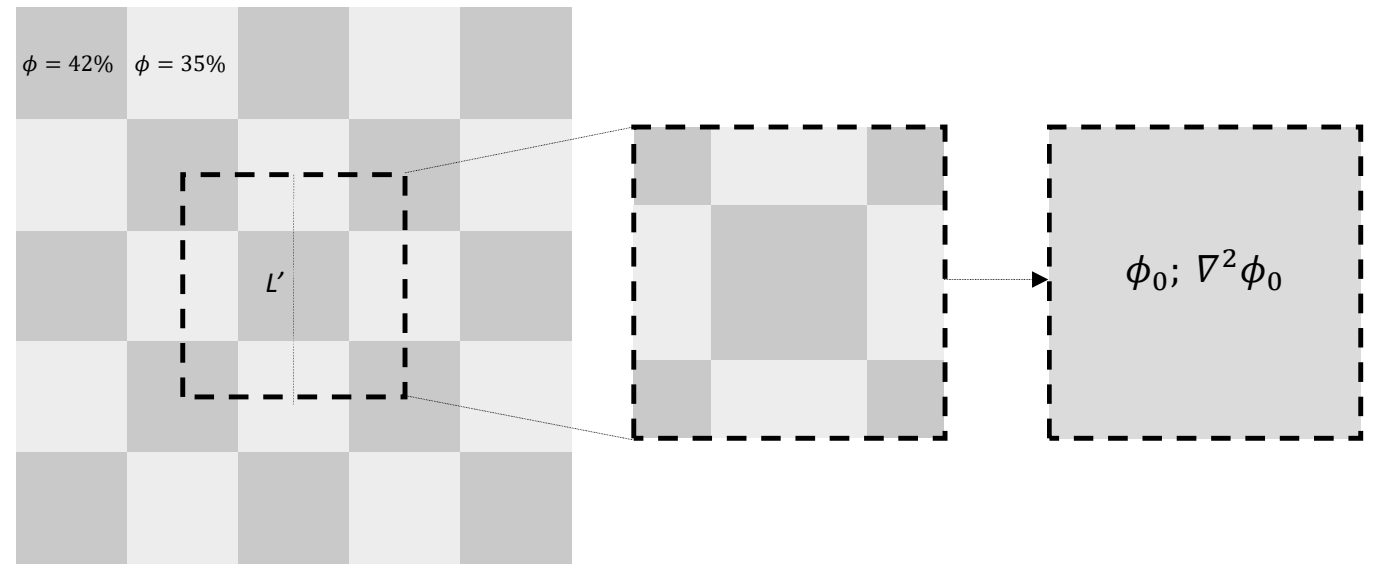

Figure 7: A schematic representation of the porosity heterogeneity. Darker and brighter areas represent high- and low-porosity zones respectively $(\phi=42 \%$ and $\phi=35 \%)$.

for the sake of simplicity, we develop a constitutive model with a unique material length $L$ (i.e. $L^{\prime}$ ), which is calibrated on specific selected zones as described in the following.

In order to evaluate $L^{\prime}$ (and consequently $L$ ), the FFT is applied on porosity profiles plotted over lines defined in different orientations crossing the main zones of the sample which have exhibited the formation of compaction bands (see Figure 8 and Figure S5 which is presented in the Supporting Information file for the sake of conciseness). The dominant wavelength obtained from the FFT analysis is the calibrated value of $L^{\prime}$.

In total, 17 lines are considered in three different planes: Lines L1, L2, L3, L4, D, E and F in a XZ cross-section; Lines L5, L6, L7, A, B and C in a YZ cross-section; Lines L8, L9, L10 and L11 defined in a XY cross-section in the middle part of the sample. These lines are intentionally chosen centered over a high porosity zone where compaction bands will form after loading. As an example, the plots over the Line L1 are presented in Figure 9. The plots concerning the lines L2 to L11 as well as the lines A to F can be found in the supporting information file (Figures S1 to S4 and S6 to S7). For all the lines, except for Lines 5, 11 and F, the dominant frequency is $0.0417 \mathrm{~mm}^{-1}$, which corresponds to a wavelength of $24 \mathrm{~mm}$. As for the lines L5, L11 and Line F, the latter value represents the second dominant wavelength. This is due to the fact that the porosity distribution is not perfectly isotropic. Nevertheless, 


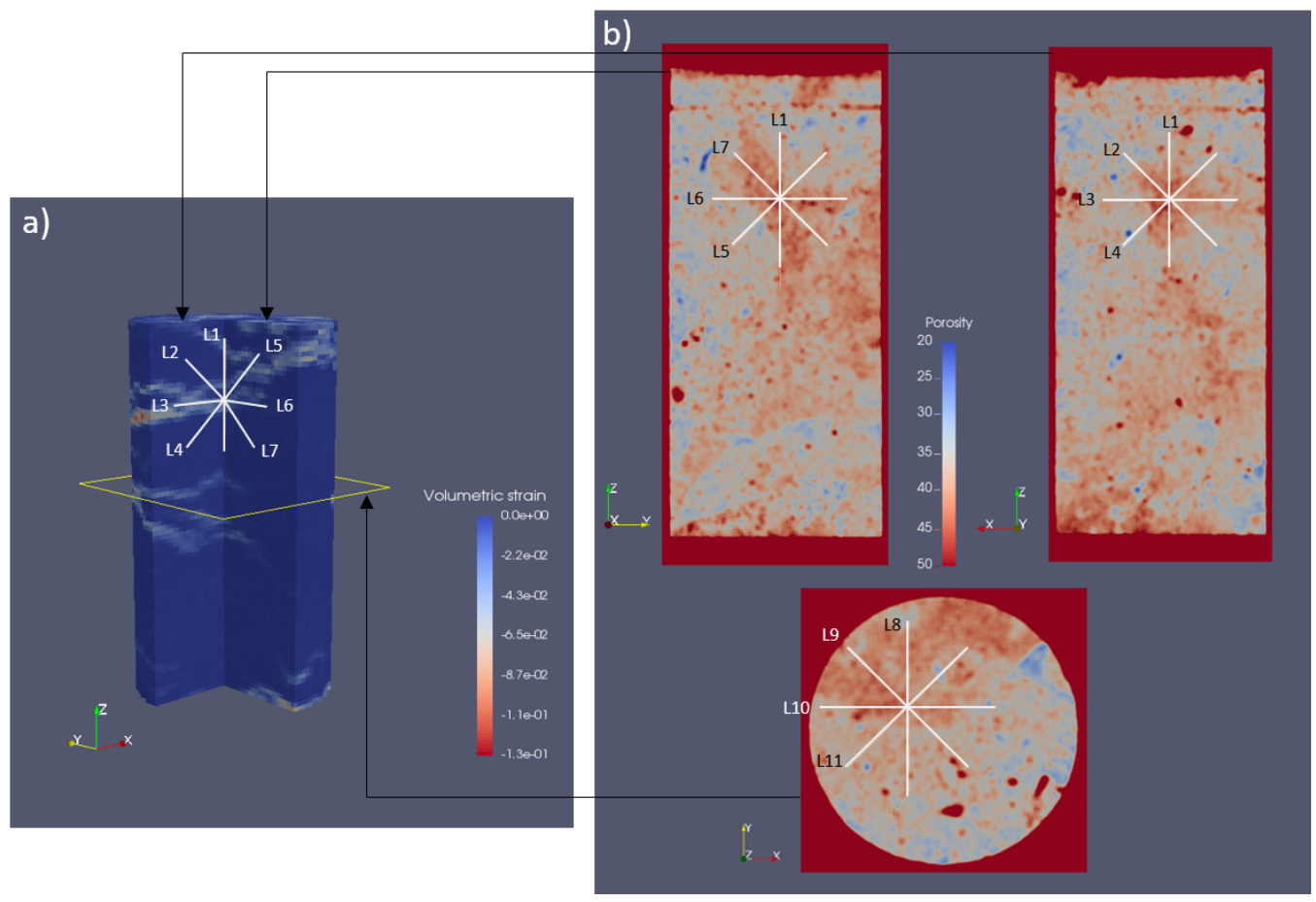

Figure 8: Definition of the lines over which porosity profiles are plotted. 
a)

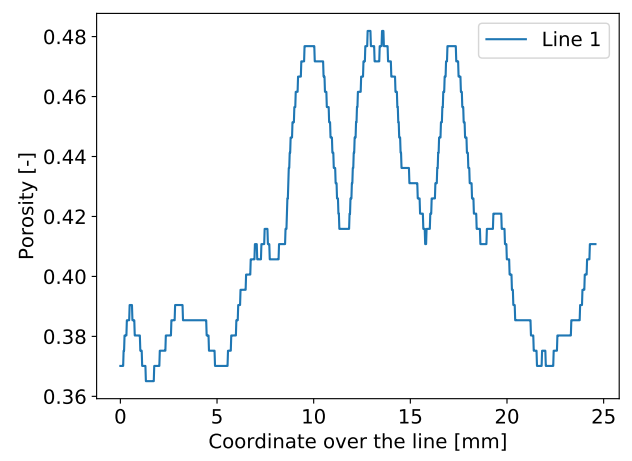

b)

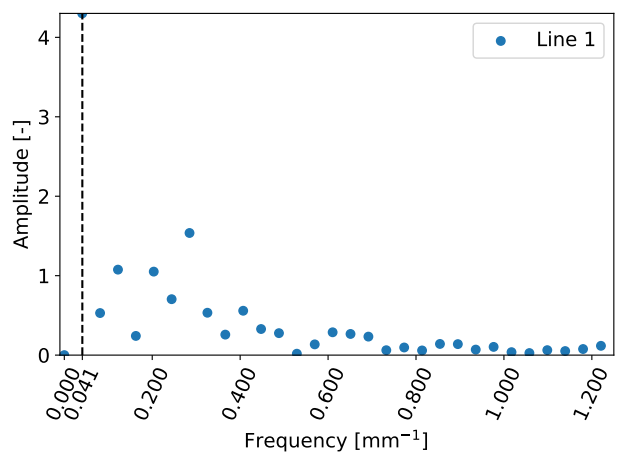

Figure 9: Porosity profile plotted over the Line 1 (a) and the corresponding FFT (b).

to keep the model simple, the material is assumed to be isotropic with a single wavelength for the porosity distribution as presented in Figure 7. Therefore, the selected material length is taken equal to $24 \mathrm{~mm}$.

\subsection{Hardening Laws}

A comparison between the classical Cauchy formulation and the enhanced gradient-dependent plasticity model, interpreted as a micromorphic continuum, is presented in Table 2. The mean value of the plastic volumetric strain, defined at the scale of the samples, is the unique internal variable in the Cauchy continuum. However, the representative elementary volume in the gradient-dependent plasticity model has a cubic shape with side length $L$ and is described by two internal variables: the porosity and its second gradient $\nabla^{2} \phi_{0}$ (whose equivalency with $\chi_{k, k}$ has been shown in Section 2.3.1). Therefore, the yield surface depends on these two variables and a method to evaluate the second gradient of porosity from experimental data is thus required.

\subsubsection{Evaluation the Second gradient of Porosity}

The computation of porosity maps from X-Ray computed tomography images has been explained in Abdallah et al. (2020). On a porosity map, a subvolume with cubic shape of side length $L$ centered on a given point is defined and the 
Table 2: Comparison between the Cauchy and micromorphic continua.

\begin{tabular}{ccc}
\hline & Cauchy continuum & Micromorphic continuum \\
\hline Constitutive law & $\dot{\sigma}_{i j}=C_{i j k l}^{e p} \dot{\epsilon}_{k l}$ & $\dot{\sigma}_{i j}=C_{i j k l}^{e p} \dot{\epsilon}_{k l}-\langle 1\rangle \frac{b_{i j}^{g} \frac{\partial F}{\partial \zeta} B L^{4}}{H_{1}} \dot{\chi}_{k, k}$ \\
& & $\dot{\zeta}=B L^{4} \dot{\chi}_{k, k}$ \\
\hline Internal variables & $\left\langle\epsilon_{v}^{p l}\right\rangle$ & $\phi_{0} ; \nabla^{2} \phi_{0} \equiv \chi_{k, k}$ \\
& $F\left(\sigma_{i j},\left\langle\epsilon_{v}^{p l}\right\rangle\right)$ & $F\left(\sigma_{i j}, \phi_{0}, \nabla^{2} \phi_{0}\right)$ or $F\left(\sigma_{i j}, \zeta, \phi_{0}\right)$ \\
\hline Yield surface & $q^{2} e^{k x}+M^{2}\left(p-p_{c}\right)\left(p+p_{t}\right)$ & $q^{2} e^{k x}+M^{2}\left(p-p_{c}\right)\left(p+p_{t}\right)$ \\
\hline Hardening laws & $\dot{p}_{c}=h_{1}\left\langle\epsilon_{v}^{p l}\right\rangle$ & $\dot{p}_{c}=\alpha_{1}\left(\dot{\phi}_{0}+\frac{L^{2}}{24} \nabla^{\dot{2}} \phi_{0}\right)$ \\
& $\dot{M}=h_{2}\left\langle\epsilon_{v}^{p l}\right\rangle$ & $\dot{M}=\alpha_{2}\left(\dot{\phi}_{0}+\frac{L^{2}}{24} \nabla^{\dot{\phi}} \phi_{0}\right)$ \\
\hline Plastic potential & $g=\beta p+q$ & $g=\beta p+q$ \\
\hline
\end{tabular}

second gradient of the porosity at the center of the subvolume is computed. It consists in fitting the porosity distribution with a second order polynomial function corresponding to the Taylor series expansion up to the second order:

$$
\phi(\underline{x}) \approx \phi_{0}+\underline{\nabla \phi_{0}} \cdot\left(\underline{x}-\underline{x_{0}}\right)+\frac{1}{2}\left(\underline{x}-\underline{x_{0}}\right)^{t} \cdot \underline{\underline{H_{0}}} \cdot\left(\underline{x}-\underline{x_{0}}\right)
$$

where $\phi_{0}, \underline{\nabla \phi_{0}}$ and $\underline{\underline{H_{0}}}$ are the porosity, its first gradient vector and its Hessian matrix respectively (with $\underline{\underline{H_{0}}}[i, j]=\frac{\partial^{2} \phi}{\partial x_{i} x_{j}}$ ), all evaluated at the center of the cube, $x_{0}$ and $\underline{x}$ are the vector positions of the center and of a random point inside the cubic domain respectively and the superscript symbol $(.)^{t}$ represents the transpose operator.

The mean value and the second order statistical moment of such distribution can be easily evaluated in terms of the central porosity $\phi_{0}$ and the second gradient $\nabla^{2} \phi_{0}=\left(\frac{\partial^{2} \phi}{\partial x^{2}}+\frac{\partial^{2} \phi}{\partial y^{2}}+\frac{\partial^{2} \phi}{\partial z^{2}}\right)_{x_{0}, y_{0}, z_{0}}$ as follows:

$$
\begin{gathered}
\langle\phi\rangle=\frac{1}{L^{3}} \iiint \phi(\underline{x}) d v=\phi_{0}+\frac{L^{2}}{24} \nabla^{2} \phi_{0} \\
\left\langle r^{2} . \phi\right\rangle=\frac{1}{L^{3}} \iiint r^{2} \phi(\underline{x}) d v=\frac{L^{2}}{4} \phi_{0}+\frac{19 L^{4}}{1440} \nabla^{2} \phi_{0}
\end{gathered}
$$

where $r=\sqrt{\left(x-x_{0}\right)^{2}+\left(y-y_{0}\right)^{2}+\left(z-z_{0}\right)^{2}}$ represents the distance of a voxel to the center of the cube. The above integrals can be computed numerically to evaluate the internal variables $\phi_{0}$ and $\nabla^{2} \phi_{0}$. 


\subsubsection{Hardening Moduli Calibration}

The yield surface is assumed to have the same expression as the one of the Cauchy formulation, with the same initial parameters presented in Table 1. Moreover, since the evolutions of the variables $p_{c}$ and $M$ are linear in terms of the mean plastic volumetric strain in the Cauchy formulation (Equation 15), it is assumed that the enhanced hardening laws are also linear in terms of the two hardening variables $\phi_{0}$ and $\nabla^{2} \phi_{0}$ :

$$
\begin{aligned}
& \dot{p}_{c}=\alpha_{1}\left(\dot{\phi}_{0}+\frac{L^{2}}{24} \nabla^{2} \phi_{0}\right) \\
& \dot{M}=\alpha_{2}\left(\dot{\phi}_{0}+\frac{L^{2}}{24} \nabla^{\dot{2}} \phi_{0}\right)
\end{aligned}
$$

as the mean plastic volumetric deformation is approximated by the mean plastic porosity change $\left\langle\epsilon_{v}^{p l}\right\rangle \simeq\left\langle\phi^{p l}\right\rangle$, which is expressed by $\dot{\phi}_{0}+\frac{L^{2}}{24} \nabla^{\dot{2}} \phi_{0}$ (see Section 3.3.1). $\alpha_{1}$ and $\alpha_{2}$ are the new hardening moduli that must be calibrated on the tests data.

The procedure followed to calibrate the moduli $\alpha_{1}$ and $\alpha_{2}$ is presented in Figure 10. For a loading stage from the State $i$ to the State $i+1$, the porosity and its second gradient are evaluated on a selected cubic domain from the porosity maps. On the other hand, the mean plastic volumetric strain are evaluated over the same domain from the DVC map using Paraview software (Ayachit, 2015). Then, from the macroscopic hardening laws given in Equations 15, the increments of $p_{c}$ and $M$ are evaluated. This procedure is repeated for different selected cubic domains inside the same sample and for several loading stages. Hence, the moduli $\alpha_{1}$ and $\alpha_{2}$ can be finally calibrated.

The sample tested under high confining pressure (10.5 MPa) (see Abdallah et al. (2020)) is considered here. Deviatoric loading has been applied in two stages: the first one is stopped just after the plasticity onset (total axial strain of $0.6 \%$ ) and it is observed that compaction bands are formed. The second loading stage is applied up to a total axial strain of $2.4 \%$, where compaction bands continue to develop. Two cubic domains, centered over high-porosity zones, are selected inside the sample as shown in Figure 11 (REV 1 and REV 2). The porosity and its second gradient are evaluated for each selected domain in the initial state and after each loading stage and are plotted in Figure 12. Both internal variables decrease (in absolute value) in due course of loading, which indicates that the material porosity becomes lower and less heterogeneous. The plastic volumetric strain is averaged over the two selected domains for each loading stage and the values are given in Table 3. Finally, the hardening moduli are calibrated as shown in Figure 


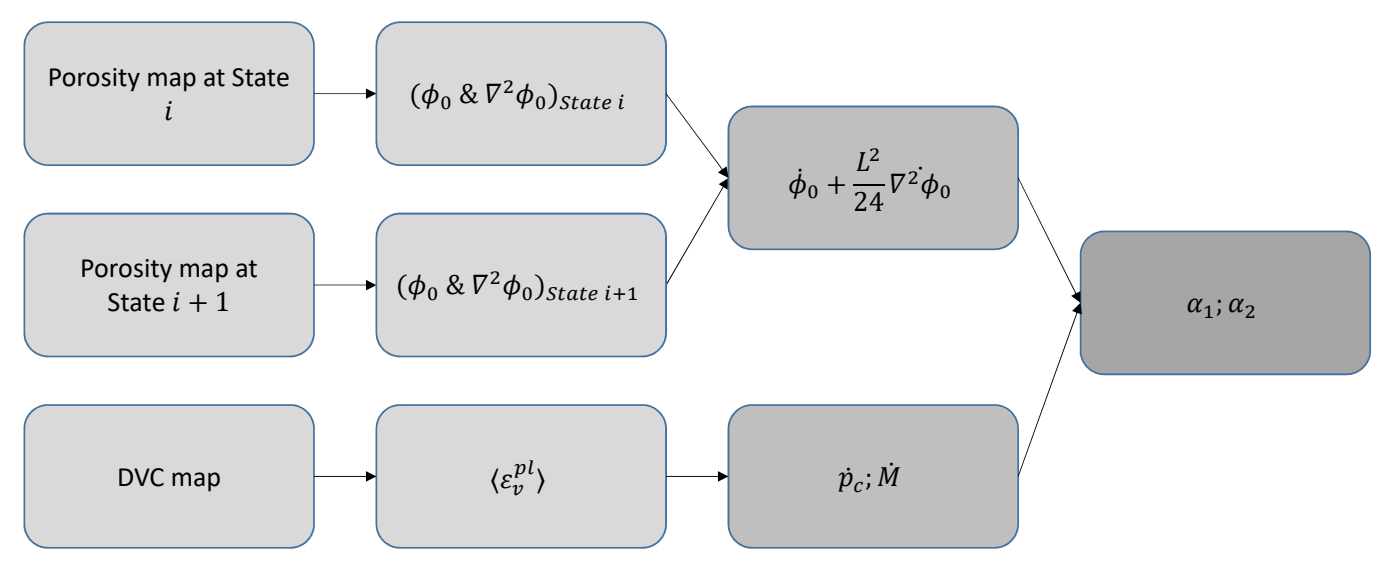

Figure 10: Procedure followed to calibrate the hardening moduli $\alpha_{1}$ and $\alpha_{2}$ for a loading stage from State $i$ to State $i+1$.

13 and the following results are obtained: $\alpha_{1}=150.9 \mathrm{MPa}$ and $\alpha_{2}=4.15$. These values are very close to those obtained for the macroscopic hardening laws $\left(h_{1}=153 \mathrm{MPa}\right.$ and $h_{2}=4.21$ in Equation 15). In other words, the mean value of the plastic porosity reduction is very close to the mean plastic volumetric deformation, which confirms that the solid matrix plastic deformation can be neglected with respect to the porosity evolution and corroborates the assumption made in Section 2.2.

Furthermore, it is important to note that both the REV 1 and REV 2 are assumed to preserve their cubic shapes when $\phi_{0}$ and $\nabla^{2} \phi_{0}$ are calculated, even in the deformed states. This assumption, which is adopted to simplify the calibration procedure, is acceptable due to the low deformation levels of the REVs (in the order of 2 to $3 \%$ as presented in Table 3 ). In addition, the high confining pressure test is sufficient to calibrate the hardening moduli, as several cubic domains can be selected inside the sample. The size of these domains is considered large enough to have the same stress state as the full cylindrical sample. Moreover, the plastic potential for this enhanced model is supposed to be identical to the one of the underlying Cauchy continuum (Equation 16). This means, that the second gradient of the porosity change is assumed to be purely elastic since $\dot{\chi}_{k, k}^{p l}=\dot{\lambda} \frac{\partial g}{\partial \zeta}=0$. 


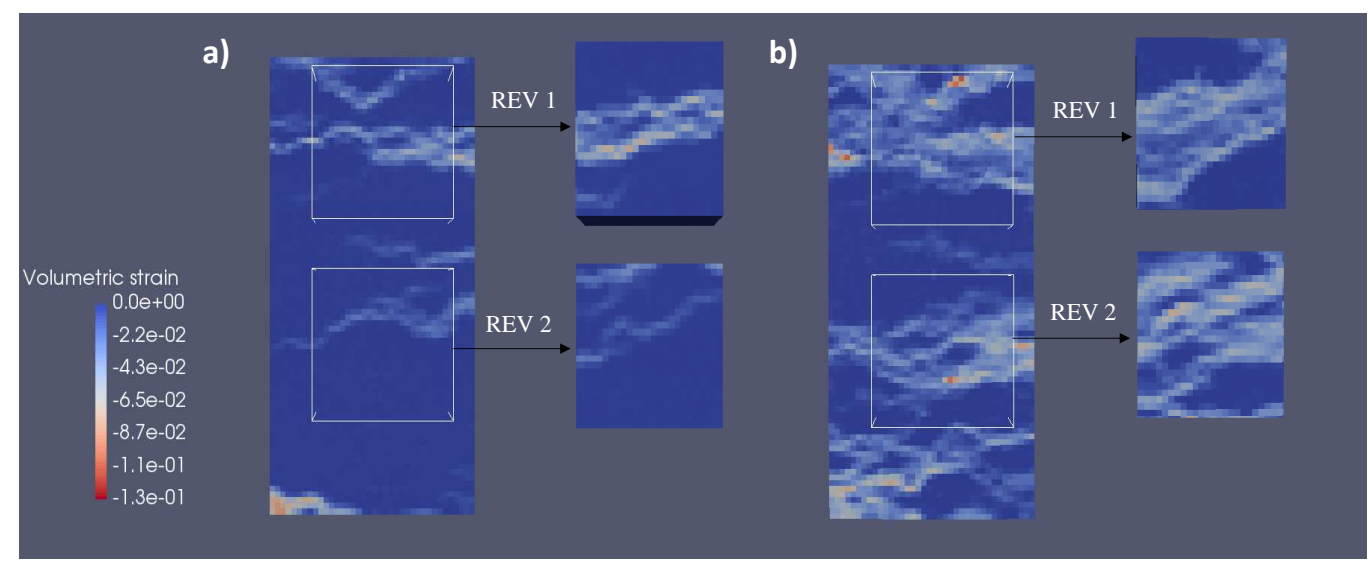

Figure 11: Two selected cubic elements, over which the mean plastic volumetric strain and the internal variables are evaluated during the a) first and b) second loading stages.

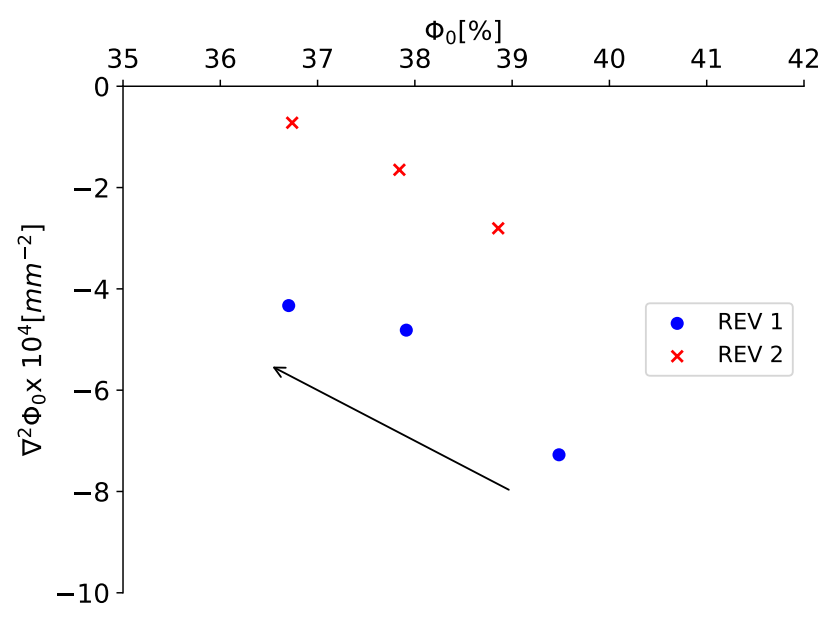

Figure 12: Evolution from the initial to the ultimate state of $\phi_{0}$ and $\nabla^{2} \phi_{0}$ for each of the two selected domains. The arrow shows the direction of the evolution with loading. 

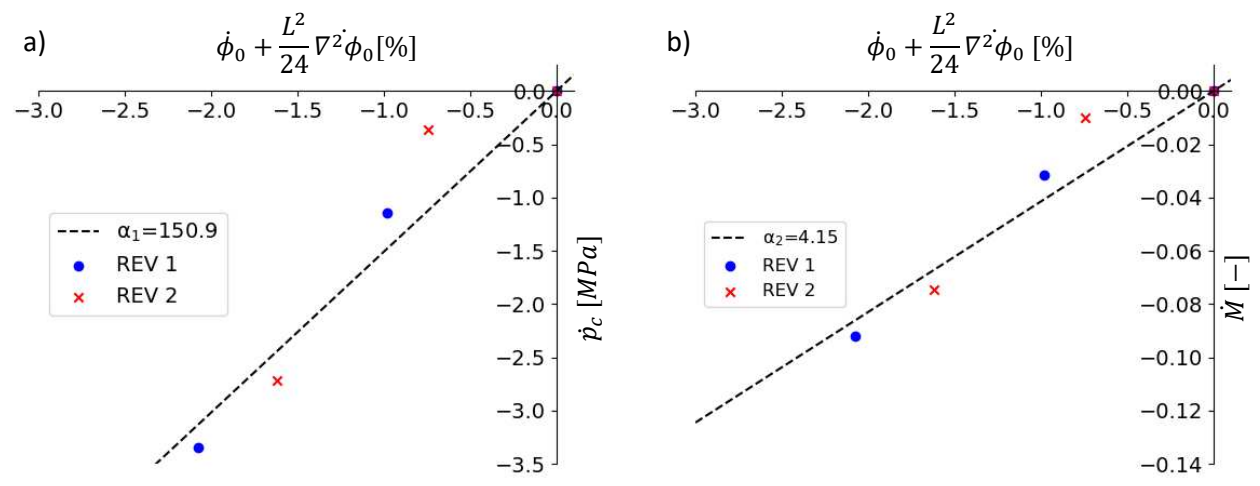

Figure 13: Evolution of $\dot{p}_{c}$ and $\dot{M}$ vs $\left(\dot{\phi}_{0}+\frac{L^{2}}{24} \nabla^{2} \phi_{0}\right)$. The dashed lines represent the fitted hardening laws obtained with the following moduli $\alpha_{1}=$ $150.9 \mathrm{MPa}$ and $\alpha_{2}=4.15$.

Table 3: Mean plastic volumetric strain for each REV evaluated in each loading stage.

\begin{tabular}{ccc}
\hline REV & Loading Stage & $\left\langle\epsilon_{v}^{p l}\right\rangle[\%]$ \\
\hline 1 & 1 & -0.74 \\
& 2 & -1.44 \\
\hline 2 & 1 & -0.24 \\
& 2 & -1.54 \\
\hline
\end{tabular}




\subsection{Elastic Modulus $B$ Calibration - Linear Stability Analysis}

To complete the calibration of the model, the higher-order elastic modulus $B$ must be evaluated. As it will be shown in the following, this parameter plays a role in the onset of compaction bands. A Linear Stability Analysis (LSA) is performed at the plasticity onset to back analyze the experiments and calibrate this parameter. In such an analysis, deformation bands are seen as the result of an instability of the homogeneous deformation process. A system is unstable in the sense of Lyapunov $(1892,1992)$ when its response to a perturbation is unbounded. The stability of homogeneous deformation is studied by considering small perturbations of the various fields and by analyzing their evolution in time (e.g. the displacement field can be written as $u=u^{0}+u^{*}$, where the superscripts ${ }^{0}$ and ${ }^{*}$ denote the homogeneous solution and the perturbation, respectively). The equilibrium equation for the homogeneous solution (Equation 6) is:

$$
\sigma_{i j, j}^{0}+\delta_{i j} \delta_{k l} \zeta_{, l k j}^{0}=0
$$

For a small perturbation around the homogeneous solution in stress and strain fields, the momentum balance can be written as follows:

$$
\sigma_{i j, j}^{*}+\delta_{i j} \delta_{k l} \zeta_{, l k j}^{*}=\rho \ddot{u}_{i}^{*}
$$

where $\rho$ is the material density. Considering the constitutive law for a small perturbation of the system (Equations 12 and 13), the momentum balance can be written as:

$$
\left(C_{i j k l}^{e}-\langle 1\rangle \frac{b_{i j}^{g} b_{k l}^{f}}{H_{1}}\right) u_{k, l j}^{*}-\langle 1\rangle \frac{b_{i j}^{g} \frac{\partial F}{\partial \frac{\zeta}{B L^{2}}} L^{2}}{H_{1}} u_{p, p k k j}^{*}+\delta_{i j} \delta_{k l} B L^{4} u_{p, p r r j k l}^{*}=\rho \ddot{u}_{i}^{*}
$$

Compaction banding is treated here as a 1D problem, although its structure can be more complex as observed in the field (Eichhubl et al., 2010). Thus, the applied perturbation to the displacement field takes the following form:

$$
u_{i}^{*}=U_{i} e^{i \frac{2 \pi}{\lambda} x_{1}+s t}
$$

with $U_{2}=U_{3}=0, \lambda$ is the wavelength of the perturbation and $s$ is the growth coefficient. Since the displacement field is continuous across the band boundaries $\left(u_{1}^{*}\left( \pm \frac{d}{2}\right)=0\right)$, the wavelength, $\lambda$, defines the band thickness: $d=\frac{\lambda}{2}$. 
The stability condition corresponds to negative values of the growth coefficient $s$ as this corresponds to pertubations which vanish in time. However, if the real part of $s$ is $\geq 0$, the system is unstable and the deformation localizes in a compaction band. After some algebraic manipulations, the following characteristic equation is obtained for the growth coefficient $s$ :

$$
-\left(\frac{2 \pi}{\lambda}\right)^{2} C_{1111}^{e p}-\left(\frac{2 \pi}{\lambda}\right)^{4}\langle 1\rangle \frac{b_{11}^{g} \frac{\partial F}{\partial \zeta} B L^{4}}{H_{1}}-\left(\frac{2 \pi}{\lambda}\right)^{6} B L^{4}-\rho s^{2}=0
$$

The above polynomial quadratic equation has two roots:

$$
s= \pm \frac{1}{L \sqrt{\rho}} \sqrt{-(2 \pi)^{2} C_{1111}^{e p} \frac{1}{\lambda^{* 2}}-\langle 1\rangle(2 \pi)^{4} b_{11}^{g} \frac{\partial F}{\partial \zeta} \frac{B L^{2}}{H_{1}} \frac{1}{\lambda^{* 4}}-(2 \pi)^{6} B \frac{1}{\lambda^{* 6}}}
$$

where $\lambda^{*}=\frac{\lambda}{L}$ is the normalized wavelength. Figure 14 a shows the plots of the growth coefficient for several confining pressures for the case of $B=0.08$ MPa. For confining pressures greater than 9.2 $\mathrm{MPa}$ (in absolute value), $s$ is observed to take positive real values, which means that compaction banding can occur. However, for lower confining pressure values, $s$ is purely imaginary indicating that compaction banding is prohibited. These stress states are illustrated by the solid line in Figure 15a. In addition, it appears that the material length does not contribute in controlling the range of confining pressures for which compaction banding can occur. The material length only scales the dominant wavelength and does not affect the range of perturbation wavelengths corresponding to positive values of the growth coefficient $s$ for a given confining pressure as shown in Figure 14b.

Accordingly, the elastic modulus $B$ is the only additional parameter that controls the range of confining pressures for which compaction banding occurs at the plasticity onset. Figure 15 shows the portion of the yield surface for which compaction bands can form for two different values of $B$. When $B$ decreases, this zone becomes larger. Based on the experimental results obtained in Abdallah et al. (2020), compaction bands are identified in the Saint-Maximin limestone sample tested under 10.5 MPa of confining pressure. However, no compaction bands are observed in the sample tested at 6 MPa of confinement, but rather compactive shear bands. In addition, Baud et al. (2017) have shown by means of acoustic emission measurements that compaction bands can form in a Saint-Maximin limestone sample tested at $9 \mathrm{MPa}$ of confining pressure in dry conditions. Assuming that the threshold 
a)

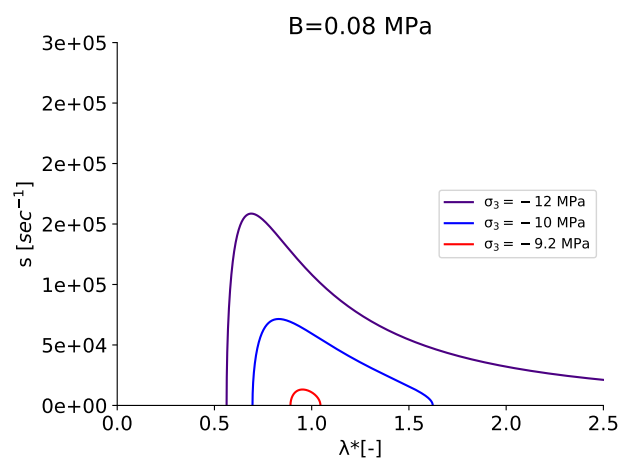

b)

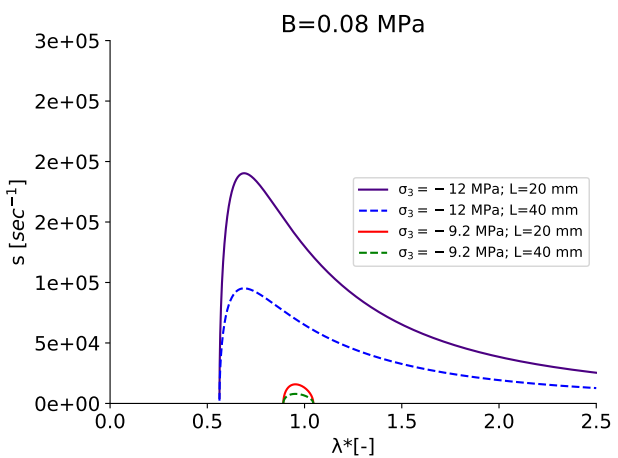

Figure 14: Growth coefficient vs the normalized perturbation wavelength under several confining pressures for $\mathrm{B}=0.08 \mathrm{MPa}\left(\rho=1600 \mathrm{~kg} / \mathrm{m}^{3}\right.$ in all cases): (a) for $L=24 \mathrm{~mm}$; (b) Effect of the material length $L$.

for the formation of compaction bands is at the mid-point of these two values (i.e. 7.5 MPa), the corresponding value of the parameter $B$ would be 0.025 $\mathrm{MPa}$ (Figure 15b), which will be retained in the following.

For the considered constitutive model and calibrated parameters, one can note that under hydrostatic compression, compaction banding is theoretically possible as the slope of the yield surface $\mu$ tends to $-\infty$ and assuming that the dilatancy coefficient is the same as for the deviatoric tests (i.e. $\beta=$ -0.75 ). For these values, the bifurcation condition (see Equation 26) in the classical formulation $(B=0)$ is fulfilled. Thus, the domain of stress states on the yield surface for which compaction banding is possible extends up to the hydrostatic axis as shown in Figure 15. However, in hydrostatic compression, no strain localization is observed in the experiments (Abdallah et al., 2020). We would need additional triaxial tests at higher confining pressures to calibrate further the dilatancy parameter which probably evolves at these high levels. 
a)

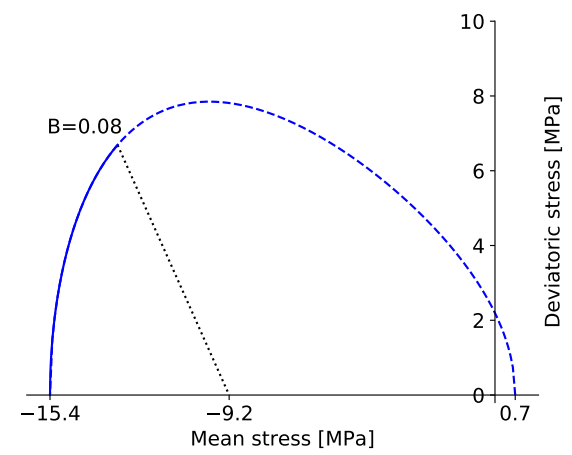

b)

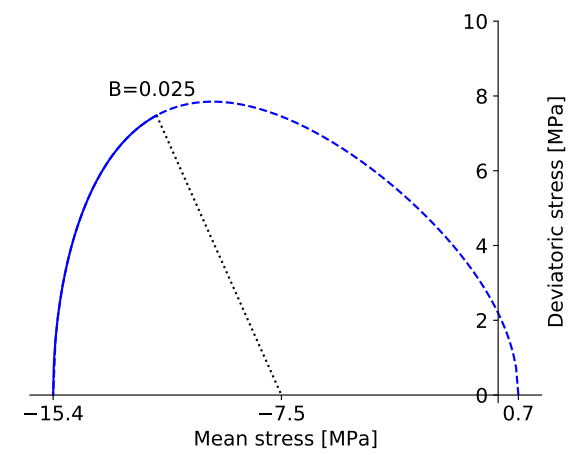

Figure 15: Range of confining pressures for which compaction bands occur (highlighted by the solid line) in the case of: (a) $\mathrm{B}=0.08 \mathrm{MPa}$; (b) $\mathrm{B}=0.025$ MPa.

\section{Numerical Implementation and Model Back- analysis}

In order to simulate the formation of compaction bands and follow their evolution, the above constitutive model is implemented in the finite element code Numerical Geolab (Stefanou, 2018) as a general micromorphic continuum of degree 2. The restrictions $u_{i, j} \equiv \chi_{i j}$ and $\chi_{i j, k} \equiv \chi_{i j k}$, presented in Section 2.3, are introduced indirectly by means of penalty moduli (E) and an incremental formulation is followed. This platform uses the Fenics library for finite element generation and the volume is meshed using tetrahedral elements with linear interpolation shape functions and using reduced integration. 1D triaxial compression simulations are performed on parallelepipedic samples with a height equal to the material length $L$. This means that a primitive cell as defined in Figure 7 is tested (i.e. one high-porosity and one low-porosity zones are stitched together). Constant confining pressure is imposed on the lateral boundaries, zero displacement and controlled axial displacement are imposed on the lower and upper boundaries respectively (Figure 16). The higher order stresses are taken equal to zero on all the boundaries. It is out of the scope of the current study to explore the model prediction for the loading cases that do not reveal the formation of compaction bands. Simulations on a sin- 


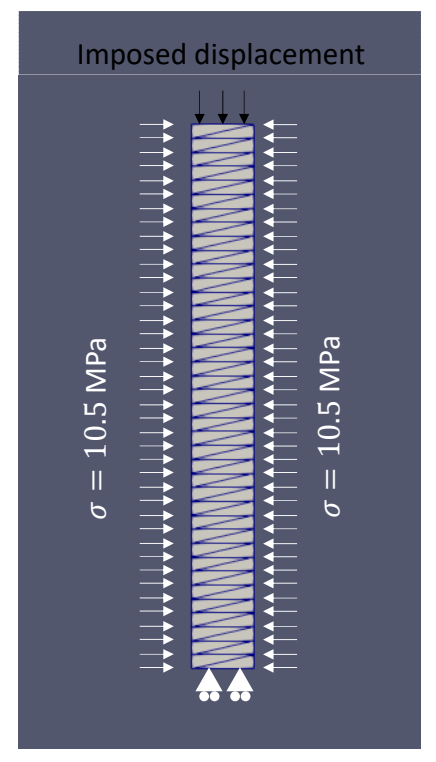

Figure 16: Schematic for the finite element model and its boundary conditions.

gle element have been first performed in order to simulate the homogeneous response and compare with experimental data. These plots are presented in the supporting information file (Figures S8 and S9). To investigate the mesh-dependency problem, numerical simulations in triaxial loading are performed for several mesh sizes: 25, 40, 50 and 70 elements along the loading direction (Figure S10 in the supporting information). No mesh-dependency is observed for a mesh size of $0.48 \mathrm{~mm}$ (50 elements) or finer. Moreover, a sensitivity analysis on the parameters $B$ and $L$ is performed and the results are shown in Figures S11 and S12 in the supporting information. The two parameters are observed to have similar impact on compaction banding, as for smaller values, compaction bands become thinner and the amount of plastic strain inside the band becomes greater.

\subsection{Triaxial Test under High Confining Pressure}

In this section, the numerical modelling of a triaxial compression loading under $10.5 \mathrm{MPa}$ of confining pressure is performed. The plastic volumetric strain maps at different levels of loading are shown in Figure 17. A material imperfection is introduced in the middle of the sample in order to trigger 
strain localization at the center: the initial value of the variable $p_{c}$ is reduced by $2 \%$ over $3 \mathrm{~mm}$ ( 6 elements). A compaction band is formed at the plasticity onset, in accordance with the experimental observations. Then, the band thickness is observed to increase with loading and the plastic strain continues to localize inside the band: $-1.3 \%$ of plastic volumetric strain is found inside the band for $-0.6 \%$ of imposed total axial strain (i.e. the level of the first loading stage applied in the experiment). The plastic strain continues to increase inside the band and $-5.6 \%$ of plastic volumetric strain is finally reached for $-2.4 \%$ of imposed total axial strain (i.e. the second loading stage in the experiment).

In order to evaluate the band thickness, the profiles of the accumulated plastic volumetric strain over the loading direction are considered (Figure 18). By analogy with the method used to evaluate a shear band thickness in Rattez et al. (2018), the compaction band thickness is estimated here by fitting a trigonometric interpolating function $f(z)$ with the vertical profiles of the plastic volumetric strain:

$$
f(z)=\frac{a_{1}}{2} \cdot \Omega_{\left[a_{2}-\frac{a_{3}}{2} ; a_{2}+\frac{a_{3}}{2}\right]}(z) \cdot\left[\cos \left(2 \pi \frac{z-a_{2}}{a_{3}}\right)+1\right]
$$

where $a_{1}$ is the maximum plastic volumetric strain, $a_{2}$ is the position of the band center, $a_{3}$ is the wavelength of the cosine function and $\Omega_{\left[a_{2}-\frac{a_{3}}{2} ; a_{2}+\frac{a_{3}}{2}\right]}(z)$ is a rectangular function defined as:

$$
\Omega_{\left[a_{2}-\frac{a_{3}}{2} ; a_{2}+\frac{a_{3}}{2}\right]}(z)=\left\{\begin{array}{lll}
1 & \text { if } & z \in\left[a_{2}-\frac{a_{3}}{2} ; a_{2}+\frac{a_{3}}{2}\right] \\
0 & \text { if } & z \notin\left[a_{2}-\frac{a_{3}}{2} ; a_{2}+\frac{a_{3}}{2}\right]
\end{array}\right.
$$

The wavelength $a_{3}$ is interpreted as the band thickness. The function $f(z)$ is successively fitted with the vertical profiles and the results are presented with black dashed lines in Figure 18. Then, the compaction band thickness evolution with respect to the imposed total axial strain is depicted in Figure 19. The initial thickness of the band at the plasticity onset is evaluated at $4.42 \mathrm{~mm}$. This thickness increases rapidly with loading to reach around 12 $\mathrm{mm}$ for $-0.3 \%$ of imposed total axial strain. Then, the band continues to expand slowly and reaches a thickness of $15.4 \mathrm{~mm}$ for an imposed total axial strain of $-2.4 \%$. 


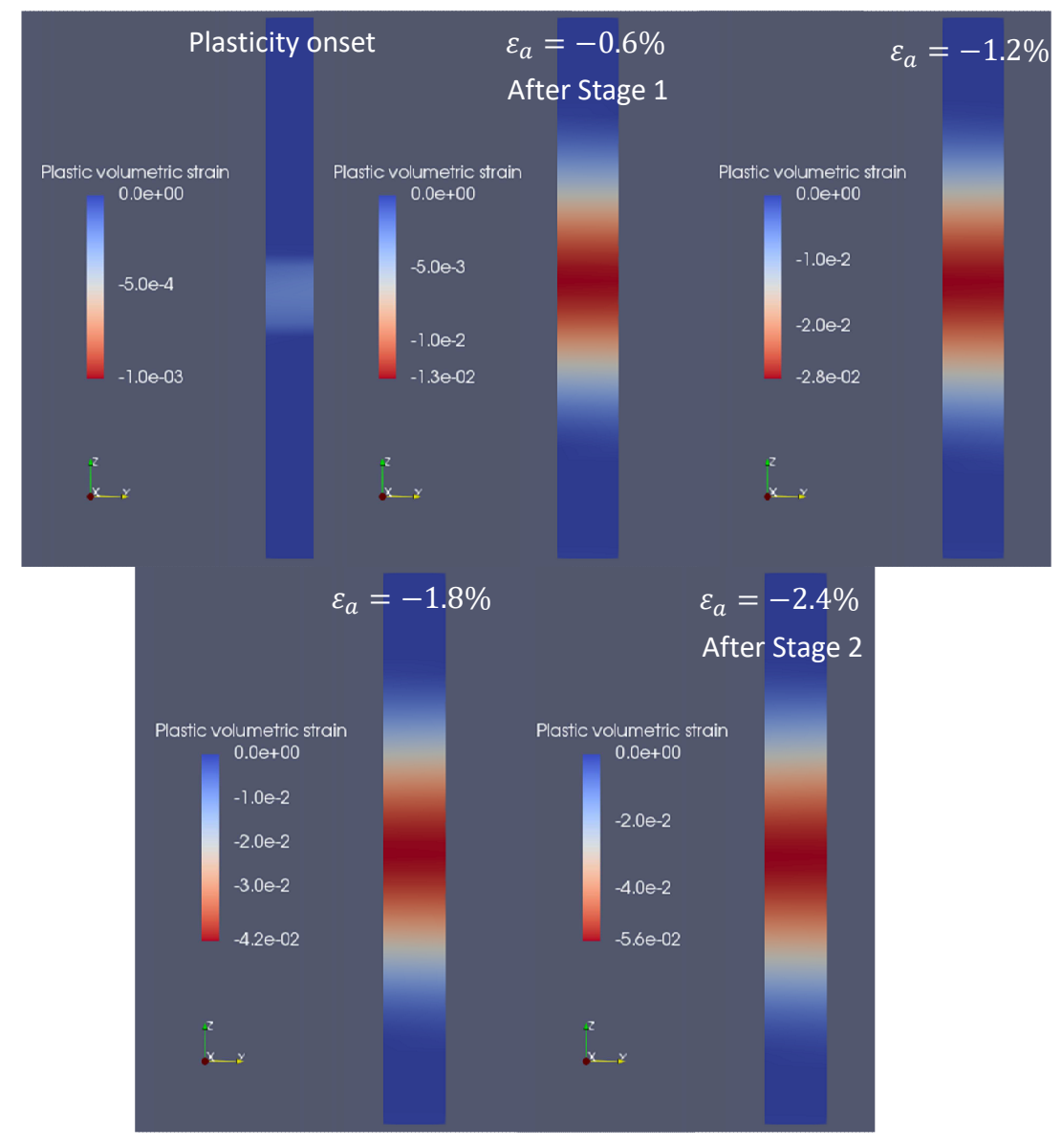

Figure 17: Accumulated plastic volumetric strain maps at different loading levels. The levels equivalent to the first and second loading stages applied in the triaxial test $(0.6 \%$ and $2.4 \%$ of total axial strain) are shown. 

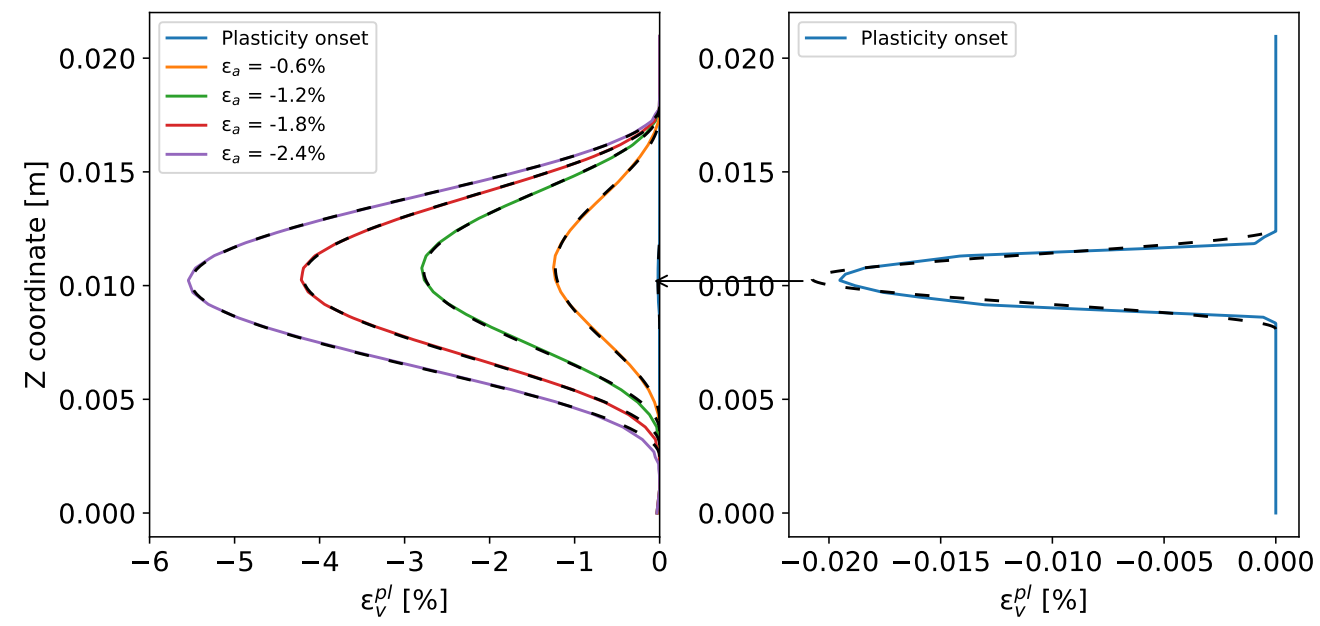

Figure 18: Vertical profiles of the accumulated plastic volumetric strain at various levels of imposed axial strain. Dashed lines represent the fitted curves $f(z)$. The plot on the right shows a zoom on the profile at the plasticity onset.

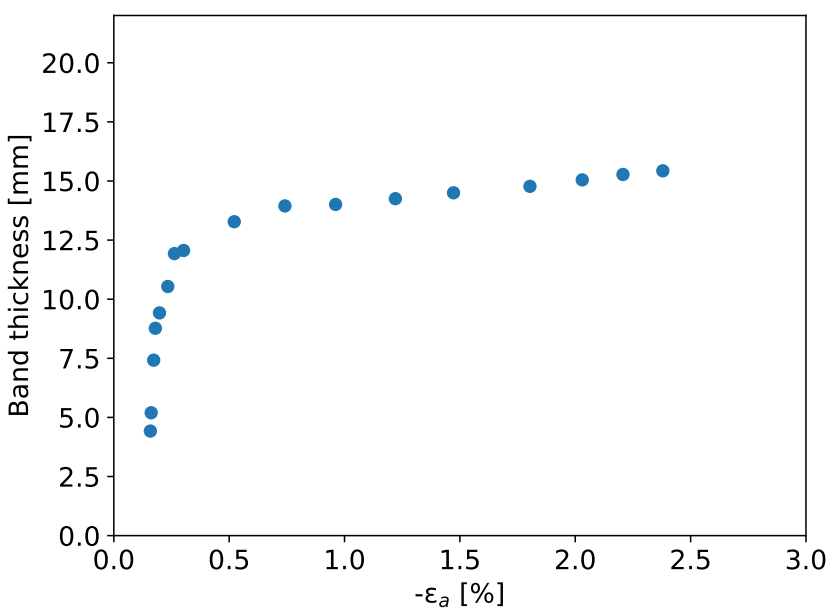

Figure 19: Compaction band thickness evolution in due course of loading. 


\subsection{Size Effect}

The size effect is explored, and two additional samples having a height $H=$ $48 \mathrm{~mm}(2 \times L)$ and $72 \mathrm{~mm}(3 \times L)$, respectively, are simulated under the same loading conditions (10.5 $\mathrm{MPa}$ of confining pressure). The same mesh size is considered for the various samples and $2.4 \%$ of total axial loading is imposed (which corresponds to the loading level applied in the experiment). The corresponding plastic axial strain maps are shown at several loading states in Figure 20. It is observed that for bigger specimens, the number of compaction bands increases, as three bands form for $H=48 \mathrm{~mm}$ (denoted by M1 to M3) and five form for $H=72 \mathrm{~mm}$ (denoted by N1 to N5). The first band to develop is located where the material imperfection is placed in the middle of the sample. This band appears at the plasticity onset whereas the other bands appear later and simultaneously at a total imposed axial strain $\epsilon_{a}^{t o t}=-0.19 \%$ in both cases $H=48 \mathrm{~mm}$ and $H=72 \mathrm{~mm}$.

The profiles of the plastic axial strain at several loading states are plotted in Figure 21. Again, the trigonometric function $f(z)$ is fitted for each band allowing to quantify the band thickness (parameter $a_{3}$ ) and the band spacing (parameter $a_{2}$ ). The band thicknesses are evaluated along loading and are compared with the case $H=24 \mathrm{~mm}$ (Figure 22). The results indicate that the band thickness is independent on the sample height and is not affected by the number of bands developed. In addition, a regular spacing between the centers of the bands is observed. This spacing is comparable to the band thicknesses, as the ratio between the band spacing over the central band thickness is close to 1 (Figure 23). Moreover, the amount of the plastic strain in the central bands in both cases $H=48 \mathrm{~mm}$ and $H=72 \mathrm{~mm}$ are similar at the same level of loading. These amounts are slightly lower than the evaluated plastic axial strain obtained in the single band formed for the case $H=24 \mathrm{~mm}$ at the ultimate loading level.

\subsection{Comparison with the Experimental Results}

The numerical modelling of the sample tested under $10.5 \mathrm{MPa}$ of confining pressure is compared to the experimental results of the sample tested under the same conditions presented in the companion paper (Abdallah et al., 2020):

1. In both experimental tests and numerical simulations, strain localization in the form of compaction bands is obtained. The bands are formed 


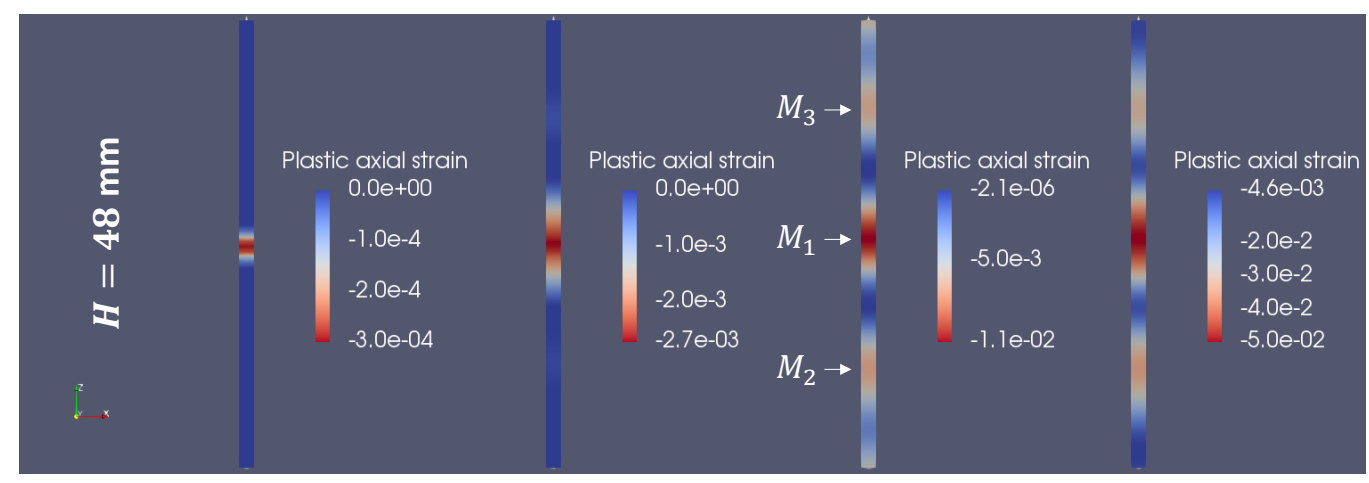

$$
\varepsilon_{a}=-0.16 \% \quad \varepsilon_{a}=-0.19 \% \quad \varepsilon_{a}=-0.60 \% \quad \varepsilon_{a}=-2.40 \%
$$

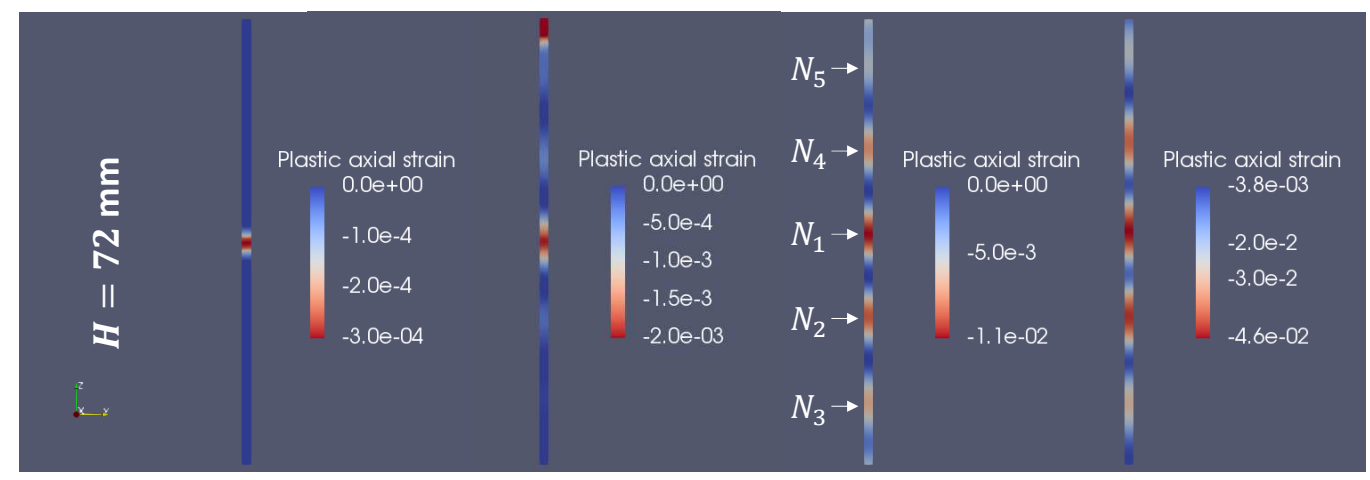

Figure 20: Accumulated plastic axial strain maps at several loading states for the cases $H=48 \mathrm{~mm}$ and $72 \mathrm{~mm}$. Three bands denoted by M1 to M3 and five bands denoted by N1 to N5 are identified for the two cases respectively. 
a)

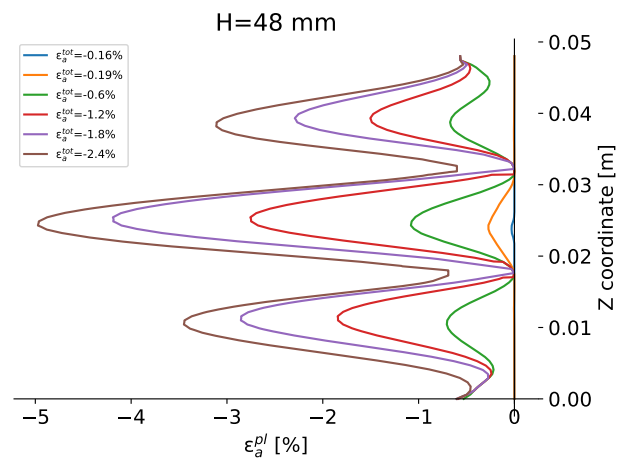

b)

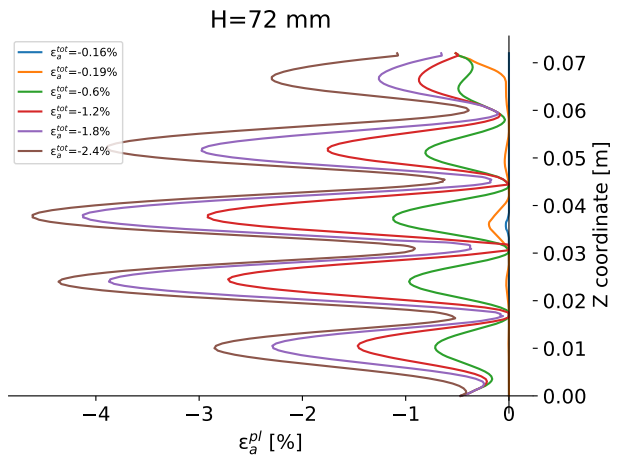

Figure 21: Accumulated plastic axial strain profiles in due course of loading for the samples of height $H=48 \mathrm{~mm}$ and $72 \mathrm{~mm}$.

a)

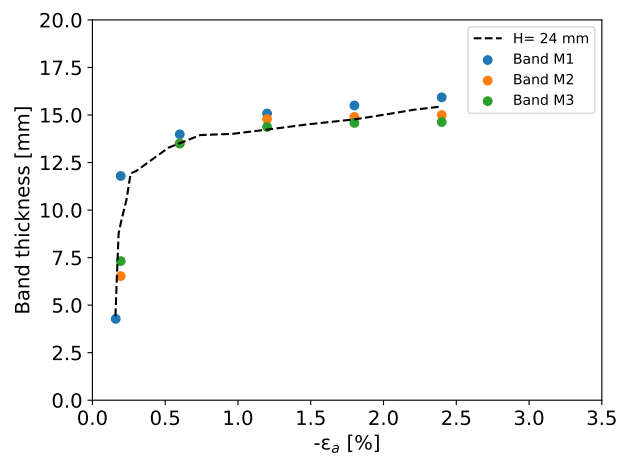

b)

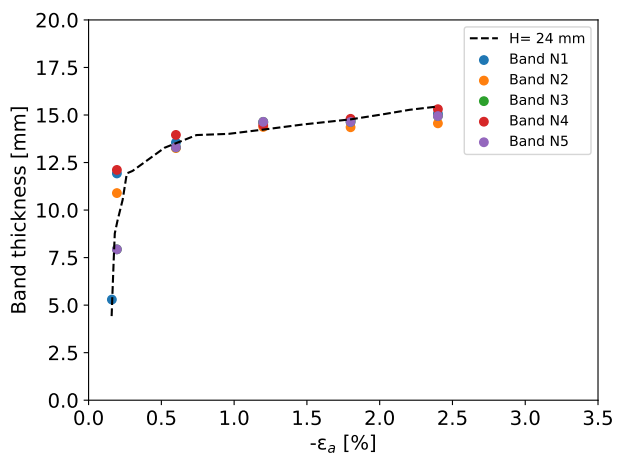

Figure 22: Band thickness evolutions in due course of loading, compared to the band thickness evolution for the sample of height $H=24 \mathrm{~mm}$ : a) Case of $H=48 \mathrm{~mm}$; b) Case of $H=72 \mathrm{~mm}$. 
a)

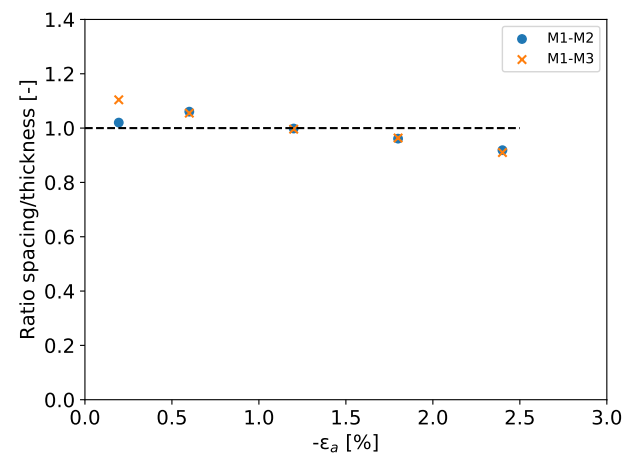

b)

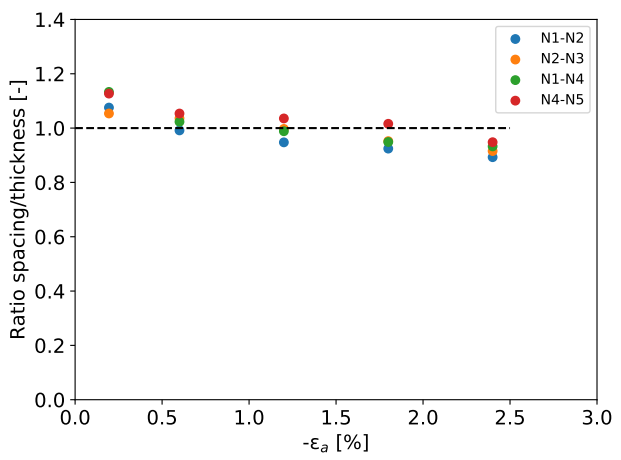

Figure 23: Ratio of band spacings over the central band thickness with respect to the imposed axial loading: a) Case of $H=48 \mathrm{~mm}$; b) Case of $H=$ $72 \mathrm{~mm}$.

at the plasticity onset.

2. Two DVC have been performed on the laboratory tested sample (Figure 11). The REV 1, over which the material length $L$ has been essentially calibrated (Section 3.2), is considered here. Then, the plastic volumetric strain in both stages is plotted over two lines defined in the normal direction to the bands (Figure 24). The obtained band thicknesses are: $8.2 \mathrm{~mm}$ after the first stage and $13.4 \mathrm{~mm}$ after the second stage. On the other hand, the thicknesses predicted in the numerical modelling are $13.0 \mathrm{~mm}$ after the first stage and $15.4 \mathrm{~mm}$ after the second loading stage.

3. The amount of the plastic volumetric strain inside the band is also investigated. The mean value of the incremental plastic volumetric strain inside the compacted zone of the REV 1 is evaluated from the profiles shown in Figure 24 (one increment corresponds to a loading stage). In addition, the profiles of the incremental plastic volumetric strain obtained in the numerical simulation are plotted and compared with the experimental data. Although the strain amount is underestimated for the first loading stage, a very good consistency between the numerical and experimental results is obtained for the second loading stage.

4. The numerical modelling of compaction banding in bigger samples have 
shown a regular spacing between the bands, which is not the case physical experiments (Figure 11). The band spacing is found to be equal to the band thickness (Figure 23), which is dependent in its turn on the material length. Since a single and uniform value for the material length has been considered in the numerical modelling, a regular spacing is obtained. Improving the model prediction would require to enhance the constitutive model by considering a distribution of material lengths that could be calibrated from the porosity maps. In the case of granular materials modelled in the framework of Cosserat theory, such an approach has been developed by considering the whole particle size distribution (Collins-Craft et al., 2020). In addition, it is worth to mention that more complex patterns of compaction bands are generally observed in laboratory tests and in the field. The development of such strain localization bands can be controlled by other parameters that are not considered in this study, such as the tectonic regime, the evolution of parameters during strain localization and the presence of fluids (Stefanou and Sulem, 2014; Soliva et al., 2016).

In conclusion, the numerical results are consistent with the experimental data in terms of the onset and development of compaction banding, the band thickness and the strain amount inside the compaction bands in due course of loading.

\section{Conclusions}

Porosity heterogenity plays a major role in controlling the formation and propagation of compaction bands in high-porosity carbonate rocks. These bands are formed in high-porosity zones, whereas denser zones remain undeformed. In addition, large pores lying inside low-porosity zones are protected by the surrounding material. This non-local character of compaction banding has lead to develop a gradient-dependent plasticity model for compaction banding in this study. Porosity and its second gradient have been identified as two independent hardening parameters. The constitutive law has been interpreted as a micromorphic continuum of degree 2, which can be seen as an extension of the second gradient theory. A back-analysis of the deformation band thickness is usually performed in order to identify the material length. In this study, a new calibration method of the additional 
a)

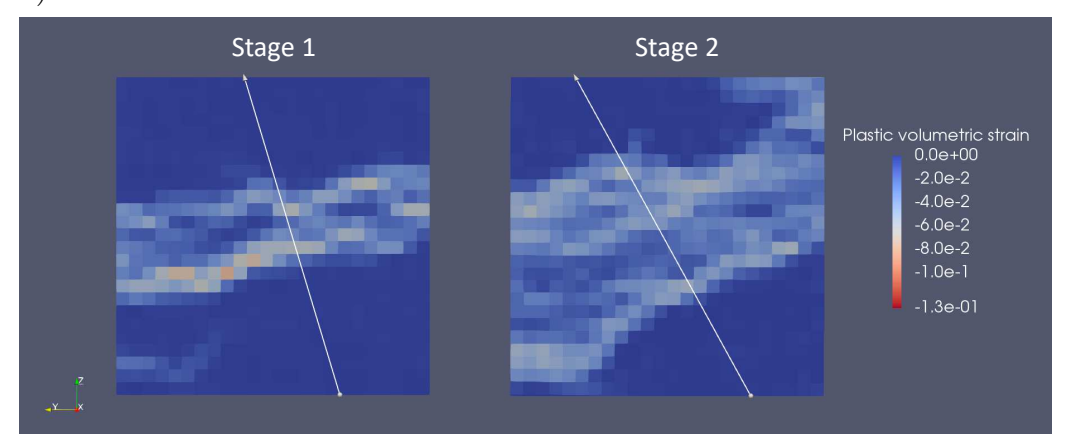

b)

c)

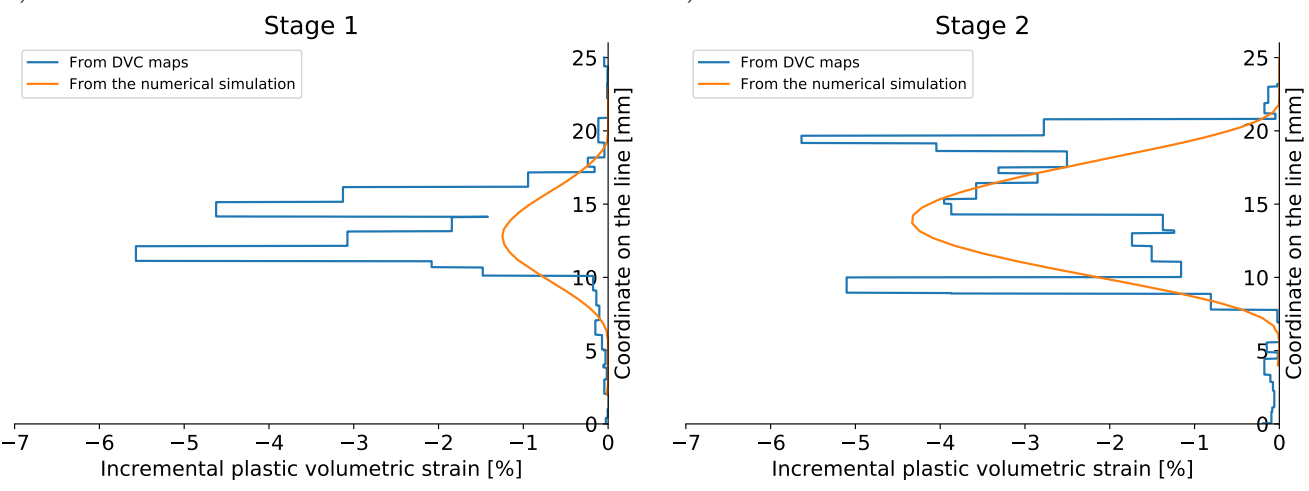

Figure 24: REV 1: Incremental plastic volumetric strain profiles. a) DVC maps; b)-c) Profiles over the lines defined on the DVC maps in the first and second stages respectively. The profiles obtained from the numerical simulation (see Figure 18) are also shown. 
parameters has been proposed based on micromechanical properties and XRay measurements without of band thickness measurements. This model and calibration approach enable a consistent simulation of the compaction banding phenomenon in high-porosity carbonate rocks.

In addition to the standard elastic-plastic parameters, the proposed model introduces several additional parameters: the material length $L$, the hardening moduli and a higher order elastic modulus $B$ linking the higher order stress with the second gradient of volumetric strain (the porosity change is identified with the plastic volumetric strain). A four-step full calibration procedure of the model has been proposed. The method is based on the macroscopic stress-strain curves, porosity and DVC maps computed at several deformation states, and on a linear stability analysis applied at the plasticity onset.

The porosity heterogeneity has been schematically represented by alternated high- and low-porosity zones of cubic shapes. The FFT applied on 1D porosity profiles has permitted to identify the wavelength of the porosity heterogeneity. This length, evaluated at $2.4 \mathrm{~cm}$, constitutes the size of the domain over which the porosity and its second gradient are evaluated. Then, two domains in which compaction bands have been experimentally observed, have been considered inside the Saint-Maximin limestone sample tested under high confining pressure. In addition, plastic volumetric strain has been evaluated over these domains which has permitted to calibrate the enhanced hardening moduli. A linear stability analysis performed on the enhanced constitutive laws has shown that the remaining elastic modulus controls the confining pressure range for which compaction bands may form at the plasticity onset. From the experimental results presented in this work and from the data provided by Baud et al. (2017), the threshold of confining pressure is estimated at $7.5 \mathrm{MPa}$. The corresponding modulus $B$ is evaluated at 0.025 MPa.

Finally, the model has been implemented in the finite element code $\mathrm{Nu}$ merical Geolab in order to validate the model with the experimental results. A sensitivity analysis of both the elastic modulus $B$ and the material length $L$ shows that when their values are smaller, compaction bands become thinner and strain localization becomes more accentuated for a given level of loading. A triaxial compression test under $10.5 \mathrm{MPa}$ of confining pressure has been thereafter simulated. The numerical results are consistent with experimental data in terms of compaction banding onset, band thickness evolution and the plastic strain amount inside the band. In addition, compaction bands 
spacing has been studied by considering bigger samples, and has been found very close to the bands thickness. This result is consistent with the linear stability analysis which considers the perturbation as a sinusoidal function.

In the proposed model, the porosity heterogeneity is simply represented by introducing one wavelength and compaction bands have been thus studied at one scale $(2.4 \mathrm{~cm})$. However, the compaction banding phenomenon has a multiscale character: one compaction band at a given scale can be seen as a zone of many compaction bands at lower scales. In this case, further analyses on the material length scale are required. Local tomography can be used in order to focus on some subdomains inside the sample along the loading process. Moreover, the proposed approach may be extended to large scale problems. From geophysical surveys, porosity maps can be constructed at larger scales and the material length of the gradient model can be assessed from the dominant wavelength. In this way, numerical computations at the scale of a reservoir can potentially be performed.

\section{A Constitutive Law Expression}

The consistency condition $\dot{F}=0$ leads to the following equation:

$$
\frac{\partial F}{\partial \sigma_{i j}} \dot{\sigma}_{i j}+\frac{\partial F}{\partial \epsilon_{v}^{p l}} \dot{\epsilon}_{v}^{p l}+\frac{\partial F}{\partial \nabla^{2} \epsilon_{v}^{p l}} \nabla^{2} \dot{\epsilon}_{v}^{p l}=0
$$

By considering the elastic law $\dot{\sigma}_{i j}=C_{i j k l}^{e}\left(\dot{\epsilon}_{k l}-\dot{\epsilon}_{k l}^{p l}\right)$ and the plastic flow rule

$\dot{\epsilon}_{k l}^{p l}=\dot{\lambda} \frac{\partial g}{\partial \sigma_{k l}}$, and by neglecting the second gradient of the elastic volumetric strain $\left(\nabla^{2} \dot{\epsilon}_{v}^{p l} \simeq \nabla^{2} \dot{\epsilon}_{v}\right)$, one can find the expression of the plastic multiplier $\dot{\lambda}$ as:

$$
\dot{\lambda}=\frac{b_{k l}^{f} \dot{\epsilon}_{k l}+\frac{\partial F}{\partial \nabla^{2} \epsilon_{v}} \nabla^{2} \dot{\epsilon}_{v}}{H_{1}}
$$

with $b_{k l}^{f}=\frac{\partial F}{\partial \sigma_{m n}} C_{m n k l}^{e}$ and $H_{1}=b_{m n}^{f} \frac{\partial g}{\partial \sigma_{m n}}-\frac{\partial F}{\partial \epsilon_{v}^{p l}} \frac{\partial g}{\partial p}$. Finally, the constitutive law is expressed as:

$$
\dot{\sigma}_{i j}=\left(C_{i j k l}^{e}-\langle 1\rangle \frac{b_{i j}^{g} b_{k l}^{f}}{H_{1}}\right) \dot{\epsilon}_{k l}+A_{i j} \nabla^{2} \dot{\epsilon}_{v}
$$

where $A_{i j}=-\langle 1\rangle \frac{b_{i j}^{g} \frac{\partial F}{\partial \nabla^{2} \epsilon_{v}}}{H_{1}}, b_{i j}^{g}=C_{i j m n}^{e} \frac{\partial g}{\partial \sigma_{m n}}$ and $\langle 1\rangle$ is the Macaulay brackets. 


\section{B Internal Power Density}

The internal power density $\delta w$ is assumed to linearly depend on these degrees of freedom and their first gradients $\left(u_{i, j}, \chi_{i j, k}\right.$ and $\left.\chi_{i j k, l}\right)$, or equivalently on $u_{i}, u_{i, j}, \eta_{i j}=u_{i, j}-\chi_{i j}, \kappa_{i j k}=\chi_{i j, k}, \eta_{i j k}=\chi_{i j, k}-\chi_{i j k}$ and $\kappa_{i j k l}=\chi_{i j k, l}$ :

$$
\delta w=K_{i} u_{i}+\sigma_{i j} u_{i, j}+s_{i j} \eta_{i j}+\nu_{i j k} \kappa_{i j k}+s_{i j k} \eta_{i j k}+\zeta_{i j k l} \kappa_{i j k l}
$$

where $K_{i}, \sigma_{i j}, s_{i j}, \nu_{i j k}, s_{i j k}, \zeta_{i j k l}$ are the dual stresses. The micromorphic medium is called as a first grade continuum of degree 2 following the nomenclature of Germain (1973). By analogy with the second gradient theory, the deformation of the subvolume and its first gradient can be identified with the deformation of the general continuum and its gradient. Two restrictions are thus considered:

1. Vanishing relative microdisplacement gradient $\eta_{i j}=0$.

2. Vanishing relative microdisplacement second gradient $\eta_{i j k}=0$.

As discussed in Germain (1973), $K_{i}$ must be zero and $\sigma_{i j}$ must be a symmetric tensor (objectivity). Taking into account these conditions and the assumed restrictions, the expression of the internal forces density can be written as:

$$
\delta w=\sigma_{i j} u_{i, j}+\nu_{i j k} u_{i, j k}+\zeta_{i j k l} u_{i, j k l}
$$

\section{Tangential Derivation Notation}

Following the definitions of Mindlin (1964), $D_{j} K_{i}$ and $D K_{i}$ are the tangential and normal derivatives of the vector field $K_{i}$ on the surface $\partial \mathscr{D}$ :

$$
K_{i, j}=D_{j} K_{i}+n_{j} D K_{i} \quad \text { with } \quad D K_{i}=n_{k} K_{i, k}
$$

Noticing that

$$
M_{i j} u_{i, j}=\left(M_{i j} u_{i}\right)_{, j}-M_{i j, j} u_{i}=D_{j}\left(M_{i j} u_{i}\right)+M_{i j} n_{j} D u_{i}-\left(D_{j} M_{i j}\right) u_{i}
$$

the application of the integration by parts method is straightforward. 


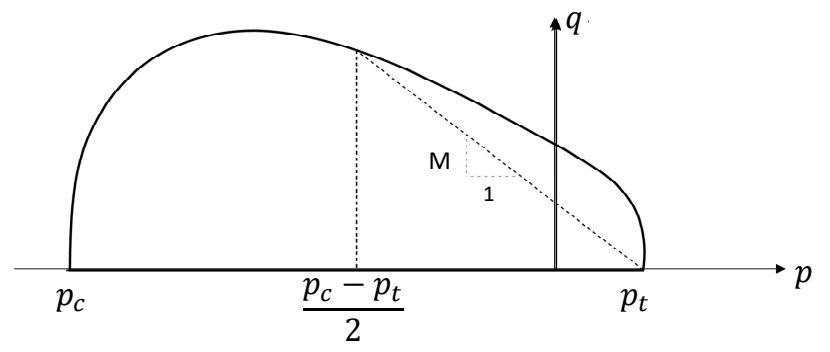

Figure 25: Schematic representation of the ACC yield surface.

\section{Asymmetric Cam-Clay Model}

The complete derivation of the ACC model is given in the Phd thesis of Samudio (2017). This model, which is an extension of the classical Modified Cam-Clay (MCC) model (Roscoe et al., 1958; Schofield and Wroth, 1968), is more flexible and allows a displacement of the hardening-softening threshold. In addition, the ACC model is better adapted to numerical implementation (see return map algorithm in Godio et al. (2016)) since its expression always takes real values and remains continuous in the real domain. Figure 25 shows a schematic representation of the yield surface, whose expression is given in Equation 14. For $p=p_{c}, x$ takes the value of 1 and for $p=-p_{t}, x$ is equal to -1 . In order to characterize the yield surface, four parameters must be evaluated: $k, M, p_{t}$ and $p_{c}$. Note that for $k=0$ and $p_{t}=0$, the MCC model is recovered.

\section{E Penalty Moduli}

The gradient-dependent plasticity model is implemented in the Numerical Geolab code as a general micromorphic continuum of degree 2. The restrictions $\eta_{i j}=0$ and $\eta_{i j k}=0$ presented in $\mathrm{B}$ are introduced indirectly, by means of two penalty moduli defined as follows:

$$
\begin{gathered}
\dot{s}_{i j}=P_{1} \dot{\eta}_{i j} \\
\dot{s}_{i j k}=P_{2} L^{2} \dot{\eta}_{i j k}
\end{gathered}
$$

For very high values of $P_{1}$ and $P_{2}$, the degrees of freedom are constrained as required. The retained values are $P_{1}=10^{9} \mathrm{MPa}$ and $P_{2}=10^{9} \mathrm{MPa}$. 


\section{Acknowledgments}

All new data included in this paper are available in the following repository: https://doi.org/10.5281/zenodo.3934361. This work has been carried out within the Labex MMCD project (Multiscale Modelling and Experimentation of Materials for Sustainable Construction) and has benefited from a French government grant managed by ANR within the frame of the national program Investments for the Future ANR-11-LABX-022-01. The numerical developments (Numerical Geolab) were supported by the European Research Council (ERC) under the European Union Horizon 2020 research and innovation program (Grant agreement 757848, CoQuake, http://Coquake.com). 


\section{References}

Abdallah, Y., Sulem, J., Bornert, M., Ghabezloo, S., Stefanou, I., 2020. Compaction banding in high-porosity carbonate rocks. Part I: Experimental observations. Journal of Geophysical Research Submitted for publication.

Ayachit, U., 2015. The paraview guide: a parallel visualization application. Kitware, Inc.

Barbier, E., 2002. Geothermal energy technology and current status: an overview. Renewable and Sustainable Energy Reviews 6, 3-65. doi:https: //doi.org/10.1016/S1364-0321(02)00002-3.

Baud, P., Schubnel, A., Heap, M., Rolland, A., 2017. Inelastic compaction in high-porosity limestone monitored using acoustic emissions. Journal of Geophysical Research: Solid Earth 122, 9989-10,008. doi:https://doi. org/10.1002/2017 JB014627.

Baud, P., Vinciguerra, S., David, C., Cavallo, A., Walker, E., Reuschlé, T., 2009. Compaction and failure in high porosity carbonates: Mechanical data and microstructural observations. Pure and Applied Geophysics 166, 869-898. doi:https://doi.org/10.1007/s00024-009-0493-2.

Bésuelle, P., Rudnicki, J.W., 2004. Localization: shear bands and compaction bands, in: International Geophysics. Elsevier, pp. 219-322. doi:https: //doi.org/10.1016/s0074-6142(03)80021-9.

Cashman, S., Cashman, K., 2000. Cataclasis and deformation-band formation in unconsolidated marine terrace sand, Humboldt County, California. Geology 28, 111-114. doi:https://doi.org/10.1130/0091-7613(2000) 28<111: CADFIU>2 . 0. CO; 2 .

Cilona, A., Baud, P., Tondi, E., Agosta, F., Vinciguerra, S., Rustichelli, A., Spiers, C.J., 2012. Deformation bands in porous carbonate grainstones: Field and laboratory observations. Journal of Structural Geology 45, $137-$ 157. doi:https://doi.org/10.1016/j.jsg.2012.04.012.

Cilona, A., Faulkner, D.R., Tondi, E., Agosta, F., Mancini, L., Rustichelli, A., Baud, P., Vinciguerra, S., 2014. The effects of rock heterogeneity on compaction localization in porous carbonates. Journal of Structural Geology 67, 75-93. doi:https://doi.org/10.1016/j.jsg.2014.07.008. 
Collins-Craft, N.A., Stefanou, I., Sulem, J., Einav, I., 2020. A Cosserat Breakage Mechanics model for brittle granular media. Journal of the Mechanics and Physics of Solids, 103975doi:https://doi.org/10.1016/j. jmps.2020.103975.

Coussy, O., 2011. Mechanics and physics of porous solids. John Wiley \& Sons.

Das, A., Nguyen, G.D., Einav, I., 2011. Compaction bands due to grain crushing in porous rocks: a theoretical approach based on breakage mechanics. Journal of Geophysical Research: Solid Earth 116. doi:https: //doi.org/10.1029/2011JB008265.

De Borst, R., Mühlhaus, H.B., 1992. Gradient-dependent plasticity: formulation and algorithmic aspects. International Journal for Numerical Methods in Engineering 35, 521-539. doi:https://doi.org/10.1002/ nme. 1620350307.

Du Bernard, X., Eichhubl, P., Aydin, A., 2002. Dilation bands: A new form of localized failure in granular media. Geophysical Research Letters 29, 29-1-29-4. doi:https://doi.org/10.1029/2002GL015966.

Eichhubl, P., Hooker, J.N., Laubach, S.E., 2010. Pure and shear-enhanced compaction bands in aztec sandstone. Journal of Structural Geology 32, $1873-1886$.

Esin, M., Dyskin, A., Pasternak, E., Xu, Y., 2017. Mode I crack in particulate materials with rotational degrees of freedom. Engineering Fracture Mechanics 172, 181-195. doi:https://doi.org/10.1016/j.engfracmech. 2016.12.024.

Forest, S., Sievert, R., 2006. Nonlinear microstrain theories. International Journal of Solids and Structures 43, 7224-7245. doi:https://doi.org/ 10.1016/j.ijsolstr.2006.05.012.

Germain, P., 1973. The method of virtual power in continuum mechanics. Part 2: Microstructure. SIAM Journal on Applied Mathematics 25, 556575. doi:https://doi.org/10.1137/0125053. 
Godio, M., Stefanou, I., Sab, K., Sulem, J., 2016. Multisurface plasticity for Cosserat materials: plate element implementation and validation. International Journal for Numerical Methods in Engineering 108, 456-484. doi:https://doi.org/10.1002/nme.5219.

Hall, S.A., Desrues, J., Viggiani, G., Bésuelle, P., Andò, E., 2012. Experimental characterisation of (localised) deformation phenomena in granular geomaterials from sample down to inter-and intra-grain scales. Procedia Iutam 4, 54-65. doi:https://doi.org/10.1016/j. piutam.2012.05.007.

Holcomb, D., Rudnicki, J.W., Issen, K.A., Sternlof, K., 2007. Compaction localization in the earth and the laboratory: state of the research and research directions. Acta Geotechnica 2, 1-15. doi:https://doi .org/10. 1007/s11440-007-0027-y.

Issen, K., Rudnicki, J.W., 2000. Conditions for compaction bands in porous rock. Journal of Geophysical Research: Solid Earth 105, 21529-21536. doi:https://doi.org/10.1029/2000JB900185.

Lyapunov, A.M., 1892. The general problem of motion stability. Annals of Mathematics Studies 17.

Lyapunov, A.M., 1992. The general problem of the stability of motion. International Journal of Control 55, 531-534. doi:https://doi.org/10.1080/ 00207179208934253.

Mindlin, R.D., 1964. Micro-structure in linear elasticity. Archive for Rational Mechanics and Analysis 16, 51-78. doi:https://doi.org/10.1007/ bf 00248490 .

Olsson, W., Holcomb, D., Rudnicki, J., 2002. Compaction localization in porous sandstone: Implications for reservoir mechanics. Oil \& Gas Science and Technology 57, 591-599. doi:https://doi.org/10.2516/ogst: 2002040.

Pamin, J.K., 1996. Gradient-dependent plasticity in numerical simulation of localization phenomena. Phd dissertation. Technische Universiteit Delft, Netherlands.

Rath, A., Exner, U., Tschegg, C., Grasemann, B., Laner, R., Draganits, E., 2011. Diagenetic control of deformation mechanisms in deformation bands 
in a carbonate grainstone. AAPG bulletin 95, 1369-1381. doi:https: //doi.org/10.1306/01031110118.

Rattez, H., Stefanou, I., Sulem, J., Veveakis, M., Poulet, T., 2018. The importance of thermo-hydro-mechanical couplings and microstructure to strain localization in $3 \mathrm{~d}$ continua with application to seismic faults. Part II: Numerical implementation and post-bifurcation analysis. Journal of the Mechanics and Physics of Solids 115, 1-29. doi:https://doi .org/10. 1016/j.jmps. 2018.03.003.

Raude, S., Giot, R., Foucault, A., Fernandes, R., 2015. An identification method to calibrate higher-order parameters in local second-gradient models. Comptes Rendus Mécanique 343, 443-456. doi:https://doi .org/10. 1016/j.crme.2015.06.002.

Roscoe, K.H., Schofield, A., Wroth, C., 1958. On the yielding of soils. Geotechnique 8, 22-53. doi:https://doi.org/10.1680/geot.1958.8.1. 22.

Rudnicki, J.W., 2007. Models for compaction band propagation. Geological Society, London, Special Publications 284, 107-125. doi:https://doi. org/10.1144/SP284.8.

Rudnicki, J.W., Rice, J., 1975. Conditions for the localization of deformation in pressure-sensitive dilatant materials. Journal of the Mechanics and Physics of Solids 23, 371-394. doi:10.1016/0022-5096(75)90001-0.

Rustichelli, A., Tondi, E., Agosta, F., Cilona, A., Giorgioni, M., 2012. Development and distribution of bed-parallel compaction bands and pressure solution seams in carbonates (Bolognano Formation, Majella Mountain, Italy). Journal of Structural Geology 37, 181-199. doi:https: //doi.org/10.1016/j.jsg.2012.01.007.

Rutqvist, J., 2012. The geomechanics of CO2 storage in deep sedimentary formations. Geotechnical and Geological Engineering 30, 525-551. doi:10. 1007/s10706-011-9491-0.

Samudio, M., 2017. Modelling of an oil well cement paste from early age to hardened state : hydration kinetics and poromechanical behaviour. $\mathrm{PhD}$ dissertation. Université Paris Est, Marne-la-Vallée, France. 
Schofield, A., Wroth, P., 1968. Critical state soil mechanics. volume 310. McGraw-Hill London.

Shahin, G., Buscarnera, G., 2020. Simulation of emergent compaction banding fronts caused by frictional boundaries. Géotechnique Letters , 1-9.

Soliva, R., Ballas, G., Fossen, H., Philit, S., 2016. Tectonic regime controls clustering of deformation bands in porous sandstone. Geology 44, 423-426.

Stefanou, I., 2018. Numerical Geolab: Multiscale Finite Elements platform for multiphysics and generalized continua.

Stefanou, I., Sulem, J., 2014. Chemically induced compaction bands: Triggering conditions and band thickness. Journal of Geophysical Research: Solid Earth 119, 880-899. doi:https://doi .org/10.1002/2013JB010342.

Sternlof, K.R., Karimi-Fard, M., Pollard, D., Durlofsky, L., 2006. Flow and transport effects of compaction bands in sandstone at scales relevant to aquifer and reservoir management. Water Resources Research 42. doi:https://doi.org/10.1029/2005WR004664.

Sulem, J., Ouffroukh, H., 2006. Shear banding in drained and undrained triaxial tests on a saturated sandstone: Porosity and permeability evolution. International Journal of Rock Mechanics and Mining Sciences 43, 292-310. doi:https://doi.org/10.1016/j.ijrmms.2005.07.001.

Vardoulakis, I., Sulem, J., 1995. Bifurcation analysis in geomechanics.

Wang, K., Sun, W., Salager, S., Na, S., Khaddour, G., 2016. Identifying material parameters for a micro-polar plasticity model via X-ray microcomputed tomographic (CT) images: Lessons learned from the curvefitting exercises. International Journal for Multiscale Computational Engineering 14, 389-413. doi:10.1615/Int JMultCompEng. 2016016841.

Zhu, W., Baud, P., Wong, T.f., 2010. Micromechanics of cataclastic pore collapse in limestone. Journal of Geophysical Research: Solid Earth 115. doi:https://doi.org/10.1029/2009JB006610. 